Article

\title{
A Polar Fuzzy Control Scheme for Hybrid Power System Using Vehicle-To-Grid Technique
}

\author{
Mohammed Elsayed Lotfy 1,2 (D), Tomonobu Senjyu 2,*, Mohammed Abdel-Fattah Farahat ${ }^{1}$, \\ Amal Farouq Abdel-Gawad ${ }^{1}$ and Hidehito Matayoshi ${ }^{2}$ \\ 1 Department of Electrical Power and Machines, Zagazig University, Zagazig 44519, Egypt; \\ mohamedabozed@zu.edu.eg (M.E.L.); farahat707@hotmail.com (M.A.-F.F.); \\ amgawad2001@yahoo.com (A.F.A.-G.) \\ 2 Department of Electrical and Electronics Engineering, University of the Ryukyus, Okinawa 903-0213, Japan; \\ e115526@yahoo.ac.jp \\ * Correspondence: b985542@tec.u-ryukyu.ac.jp; Tel.: +81-98-895-8686
}

Received: 12 May 2017; Accepted: 27 June 2017; Published: 25 July 2017

\begin{abstract}
A novel polar fuzzy (PF) control approach for a hybrid power system is proposed in this research. The proposed control scheme remedies the issues of system frequency and the continuity of demand supply caused by renewable sources' uncertainties. The hybrid power system consists of a wind turbine generator (WTG), solar photovoltaics (PV), a solar thermal power generator (STPG), a diesel engine generator (DEG), an aqua-electrolyzer (AE), an ultra-capacitor (UC), a fuel-cell (FC), and a flywheel (FW). Furthermore, due to the high cost of the battery energy storage system (BESS), a new idea of vehicle-to-grid (V2G) control is applied to use the battery of the electric vehicle (EV) as equivalent to large-scale energy storage units instead of small batteries to improve the frequency stability of the system. In addition, EV customers' convenience is taken into account. A minimal-order observer is used to estimate the supply error. Then, the area control error (ACE) signal is calculated in terms of the estimated supply error and the frequency deviation. ACE is considered in the frequency domain. Two PF approaches are utilized in the intended system. The mission of each controller is to mitigate one frequency component of ACE. The responsibility for ACE compensation is shared among all parts of the system according to their speed of response. The performance of the proposed control scheme is compared to the conventional fuzzy logic control (FLC). The effectiveness and robustness of the proposed control technique are verified by numerical simulations under various scenarios.
\end{abstract}

Keywords: hybrid power system; supply balance; frequency control; V2G; polar fuzzy; minimal-order observer

\section{Introduction}

The costs of supplying energy for remote communities such as isolated islands are usually very high. Therefore, diesel generators are frequently used to generate locally the required electricity [1]. Diesel fuel has several drawbacks: It is expensive due to adding extra cost for transportation to remote areas. In addition, engine exhaust emits some harmful gases to the environment such as sulfur oxide and carbon dioxide [2,3]. Moreover, the efficiency of diesel generators decreases dramatically for low load factor operating conditions especially below $40-50 \%$ of their capacity $[4,5]$. Furthermore, nuclear power has been used for many power systems especially in Japan. However, after the recent Fukushima disaster in 2011, many have advocated against the use of nuclear power [6]. To solve these matters, renewable power plants such as WTG and PV that are available natural and clean resources should be introduced. The penetration of WTG and PV into the Japanese power system is rising due to two main factors: improved generation efficiency of WTG and PV modules and governmental subsides [7]. Moreover, the fuel-cell (FC) is one of the important future sources for the 
green power [8]. An aqua-electrolyzer (AE) is utilized to generate hydrogen. Then, the generated hydrogen is compressed, stored in hydrogen tanks and used as fuel for FC. However, the nature of renewable sources such as WTG and PV which changes quickly and no one can predict it [9], leads to stability problemes for the hybrid power system, especially isolated ones. Due to these fluctuations in WTG and PV output powers, mismatch in the vital balance between demand and generation appears which leads to frequency and voltage fluctuations [10]. This action affects negatively the stability of the power system. So, a robust control scheme must be implemented for the power of the renewable sources to guarantee the power system performance.

To improve the stability and performance of the system, some energy storage devices such the flywheel (FW), BESS and the ultra-capacitor (UC) are often used [11]. These work as backup devices that injects power to the grid in case of excess demand and stores excess power when the generation is more than the demand. This helps to keep the secure performance of the system and balance the demand and supply sides. Therefore, it ensures acceptable levels of system frequency deviations [12,13]. FW stores electric power in the form of kinetic energy. There are many merits of FW such as high conversion efficiency of $80-90 \%$, high stored energy density, high power exchange with the system, long lifetime and also free from pollution effects [14]. In addition, UC offers another option to smooth strong and short-time power oscillations of the hybrid power system for meeting the demand thanks to its fast power response, high energy density, flexible and modular structure [15]. Furthermore, BESS is growing rapidly in the technologies of energy storage. One of The deficit of BESS is its installation cost especially for a large one. The system cost of a small power system will increase significantly if a large BESS has to be installed, which will make the whole system unfeasible from in economic terms. For this reason, the application of customers' appliances in the control of the power system instead of BESS has appeared [16]. One example of such applications is V2G, that is a concept of controlling the charging and discharging criteria between the power system and some of EVs trying to reach a balance between the generation and demand sides to suppress the system frequency fluctuations.

Recently, there have been some studies dedicated to frequency control approaches for the hybrid power system using FLC [17-21], $\mu$ synthesis scheme [22], $H_{\infty}$ and $\mu$-synthesis approach [23], neuro-fuzzy control [24], FLC with the particle swarm optimization (PSO) algorithm implementation [25,26], FLC with chaotic PSO [27], PSO with mixed $H_{2} / H_{\infty}$ control [28], the quasi-oppositional harmony search algorithm (QOHSA) [29], sliding mode control (SMC) [30], multiple model predictive control (MMPC) [31], multi-variable generalized predictive control (MGPC) [32] and Type-2 FLC with the modified harmony search algorithm (MHSA) [33] with promising results. Despite this, this research tries to mitigate supply error and modify system frequency in the face of renewable suppliers' uncertainties and random load fluctuations to face the deficits associated with most of these previous techniques, such as $H_{\infty}$ and FLC schemes. The weighting functions in the $H_{\infty}$ design process cannot be chosen in an easy way. This action affects dramatically the design process. Moreover, the $H_{\infty}$ controller order is the same as that of the plant. This produces a complicated frame which is not easy to be implemented especially for large systems. Moreover, accurate and sufficient knowledge base affects greatly the impact of the FLC scheme. Increasing the number of rules in the knowledge base leads to increase complexity, which in turn affects the computational time and requirements of memory [34]. In addition, because many states in the system can not be measured, effective estimation technique must be used for the supply error of the hybrid system in an urgent manner. Automatic generation control of only two interconnected hydro-thermal system using polar fuzzy (PF) is presented in [35] without using any renewable sources, and nothing has been mentioned about the frequency domain management. Moreover, the dynamic operation of the hybrid wind-PV-based system using the general regression neural network (GRNN) with PSO [36] and the Elman neural network (ENN) criteria [37] to extract the maximum powers from wind and PV energy sources are discussed. The lack of an effective estimation technique to overcome the drawback of requiring a substantial amount of training based on predicted scenarios associated with neural 
networks is the main deficit of these approaches. In addition, FLC and the wavelet transformer-based approach of a tracked bulldozer is intended in [38] using the battery and ultra-capacitor without either utilizing any type of renewable sources or implementing supply error control. A fuzzy polar power system stabilizer (FPPSS) using frequency domain methods is expressed in [39] without taking the variable nature of the renewable sources and the energy storage systems into consideration. An novel intelligent damping controller (NIDC) is analyzed in [40] for the static synchronous compensator (STATCOM) of a hybrid system. This scheme applies the functional link-based novel recurrent fuzzy neural network (FLNRFNN) to reduce the power fluctuation and support voltage without either considering frequency control approach or using the estimation technique to overcome the deficits of neuro-fuzzy schemes. A hybrid bird-mating optimization approach is applied in [41] to mitigate the voltage imbalance and deviation for distribution transformers of the hybrid power system without analyzing its effect on frequency control. Multiple PV grid-connected inverters with faults' analysis are presented in [42] using the inverter matrix impedance current vector (IMICV) technique. Current limit of distributed generators (DGs) is considered in this study. The limitation of the inverters current is one- to two-times the rated current. Unsymmetrical fault analysis with hybrid compensation of DGs for both islanded and grid-connected modes are discussed in $[43,44]$. The microgrid distribution ground fault (MGDGF) analysis approach is intended in [45]. The scheme analyzes the model of the ground fault for BESS in grid-connected mode in addition to islanded one. A non-directional protection scheme for detecting ground faults is analyzed in [46]. Almost all of the previous researches can not have adequate performance for different operating conditions and only they can get enough response for a specified operating point. This action is due to using small-signal simplified model. This problem is solved in the intended scheme even with using the same simplified model due to expressing the area control error (ACE) in the frequency domain and distributing the mission to balance the error among all parts of the system according to their response speed so as to cover all the frequency space of ACE are the key issues to guaranteeing the ability of the intended scheme to withstand variable scenarios and to defeat the previous researches' troubles. Moreover, the system performance with the implication of the intended approach is validated under various scenarios such as the sudden increase/decrease of wind speed, solar radiation, load demand, actual wind speed and solar radiation data and system parameters' variation to confirm its effectiveness and robustness.

For all of these previous reasons, this paper presents a new minimal-order observer-based PF control scheme for the hybrid power system. The proposed system consists of WTG, PV, the solar thermal power generator (STPG), the diesel engine generator (DEG), AE, FC, UC and FW. Moreover, a modern approach of V2G control is utilized for batteries of EVs, so it can be utilized as a tantamount large-scale BESS that helps to decrease the total cost of the power system dramatically. Furthermore, EV customers' comfort is considered. The minimal-order observer is applied to estimate the supply error. Then, ACE is calculated using the estimated supply error and the frequency deviation. ACE is counted in the frequency domain. Two PC controllers are presented in the power system. The role of each controller is to suppress one frequency component of ACE. The two inputs of the first controller are the low-frequency component of ACE and its variation with time, respectively; while the output control signal is fed to FC, EV and DEG, that have long time constants, to mitigate the low-frequency component of ACE. The high-frequency component of ACE and its change with time are two inputs for the second controller, and its output control signal is sent to UC and FW, that have already short-time constants to control the high-frequency part of ACE. The performance of the presented control scheme is compared to the conventional FLC. The numerical simulations in the MATLAB ${ }^{\circledR} /$ SIMULINK $^{\circledR}$ (Release 2016a) environment confirm the effectiveness and robustness of the intended method. The remaining parts of this research are organized as follows: Section 2 explains the hybrid power system model. The intended PF-based controllers' design scheme is discussed in Section 3, including the minimal-order observer implementation with a brief introduction of PF principles. The simulation results with detailed analysis of four scenarios is presented in Section 4. Then, the conclusion is presented in Section 5. 


\section{Hybrid Power System Configuration}

Figure 1 presents the single line diagram associated with the hybrid power system under study. The system consists of PV, STPG, AE, FC, UC, FW, DEG, EV and three units of WTG. The system block diagram is then shown in Figure 2. $P_{d}, P_{P V}, P_{W T G}, P_{S T}, P_{A E}, P_{F C}, P_{U C}, P_{F W}, P_{E V}, P_{L}$ and $P_{C}$ are the output power of DEG, PV, WTG, STPG, AE, FC, UC, FW, EV, load demand and the combined power, respectively. This paper picks up some ideas from [47], and it is a continuation of the research on the hybrid power system control techniques.

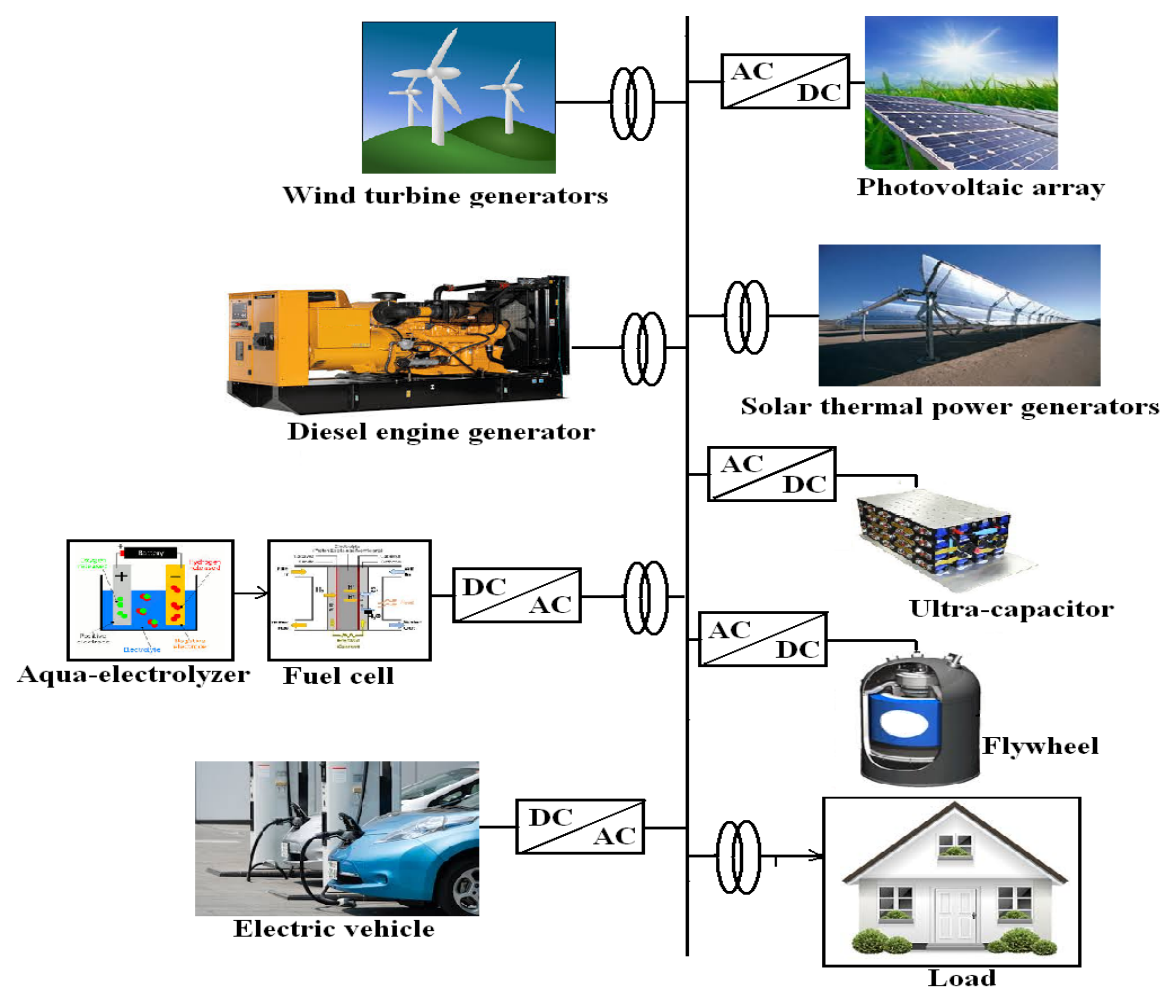

Figure 1. Single line diagram of the hybrid power system.

\subsection{Wind Turbine Generator Model}

The output power of WTG is variable according to the wind speed at every moment. The part of the wind power that is converted to mechanical power, $P_{w}$, can be expressed as follows:

$$
P_{w}=\frac{1}{2} \times C_{p} \times V_{w}^{3} \times \rho \times A
$$

where $R(=23.5 \mathrm{~m})$ is blades radius, $A=\pi R^{2}$ is rotor swept area, $\rho\left(=1.25 \mathrm{Kg} / \mathrm{m}^{3}\right)$ is air density, $C_{p}$ is aerodynamic factor and $V_{w}$ is the wind speed. $C_{p}$ is a function of both blade pitch angle $\beta$ and tip speed ratio $\lambda$. $\lambda$ can be discussed as the ratio of the speed at the blade tip compared to the wind speed that can be formulated as:

$$
\lambda=\frac{R \times \omega}{V_{w}}
$$

where $\omega(=3.14 \mathrm{rad} / \mathrm{s})$ is rotational speed of blades. Hence, an approximate expression for $C_{p}$ can be presented as $[14,47]$ :

$$
C_{p}=(0.44-0.0167 \beta) \sin \left(\frac{\pi(\lambda-3)}{15-0.3 \beta}\right)-0.0184(\lambda-3) \beta
$$




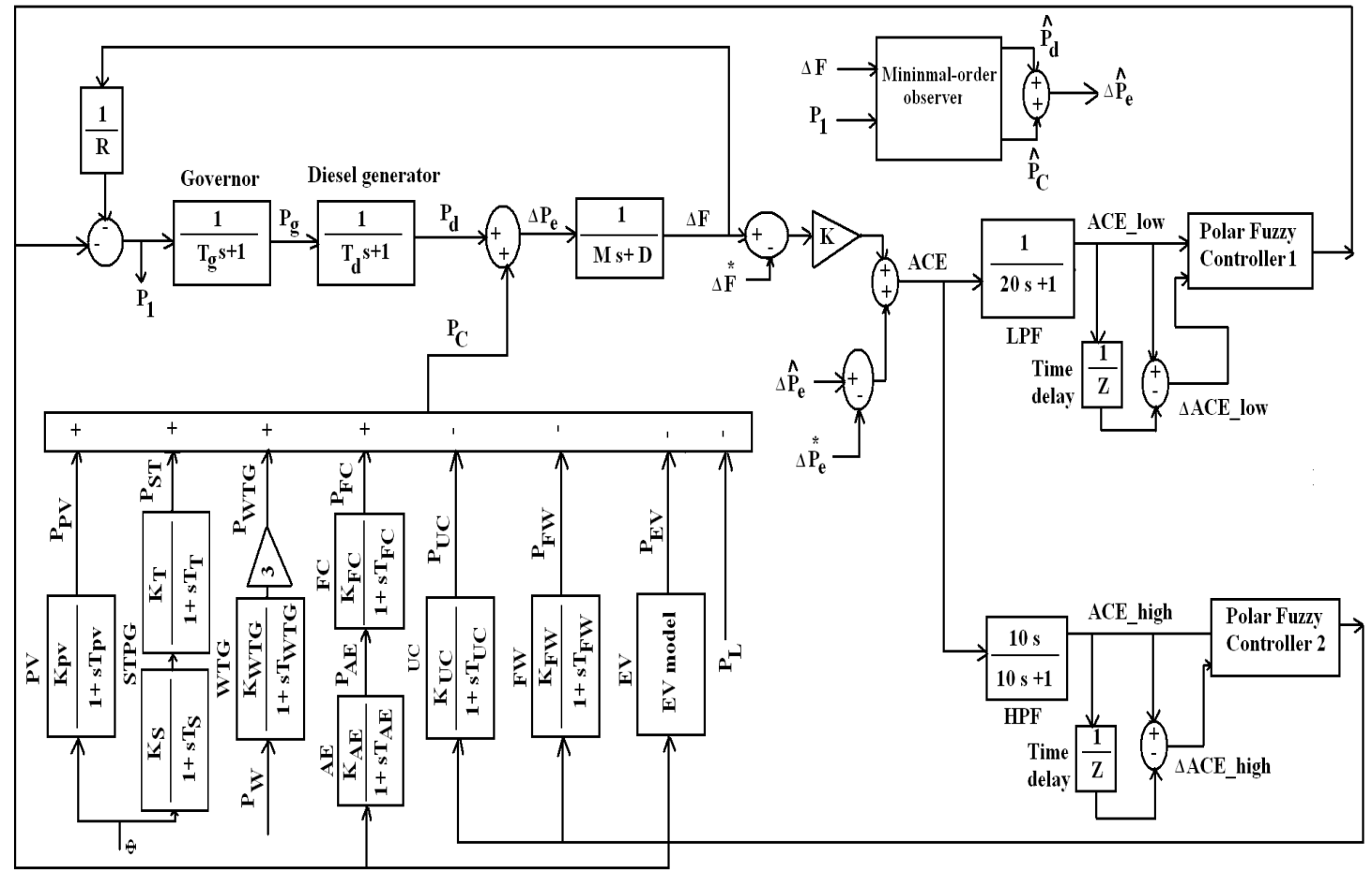

Figure 2. Block diagram of the proposed system. ACE, area control error.

\subsection{Photovoltaic Model}

The output power of the studied PV system can be expressed as:

$$
P_{p v}=\eta \times S \times \phi \times\left(1-0.005\left(T_{a}+25\right)\right)
$$

where $\eta$ is PV array efficiency ranging from $9-12 \%, \phi$ is solar radiation, $S$ is measured area of PV array and $T_{a}$ is temperature in Celsius. Value of $P_{p v}$ depends only on $T_{a}$ and $\phi$ because $S$ and $\eta$ are fixed. However, in this research, $T_{a}$ is fixed at $25^{\circ} \mathrm{C}$, and $P_{p v}$ is varied only with $\phi[10,47]$.

\subsection{Solar Thermal Power Generator Model}

Nowadays, STPG has attracted much attention all over the world. There are two main types of STPG explored by researchers: parabolic trough solar power plant (PTSPP) and also solar tower power plant (STPP). PTSPP consists of large parabolic trough solar collectors, which collect sun light and direct it to heat working fluid in the absorber tubes up to a certain temperature $400-600{ }^{\circ} \mathrm{C}$. Then, heat is extracted using heat transfer equipment to get steam, which will be used to drive the steam turbine to generate electricity. Hence, the extracted heat energy can be passed through the thermal storage medium for the later use after sunset. There are three major thermal storage mediums: molten salt, water and high temperature oil. Molten salt is still believed as the most effective thermal energy storage in economic terms. Moreover, STPP includes sun-tracking mirrors, known as heliostats that reflect the sunlight onto the tower-mounted heat exchanger. The remaining part of the steam production and electric power generation is the same as PTSPP. The main advantage of STPP over PTSPP is its higher concentration of the sun rays in which the working fluid temperature can reach to $500-1000{ }^{\circ} \mathrm{C}$. The basic model of STPG is presented in this paper as a simple transfer function as follows $[29,48]$ :

$$
G_{S T P G}(s)=\left(\frac{K_{s}}{1+s T_{s}}\right)\left(\frac{K_{T}}{1+s T_{T}}\right)
$$


where $K_{s}$ and $K_{T}$ are the gains of the STPG, whereas $T_{s}$ and $T_{T}$ are the time constants of the STPG.

\subsection{Ultra-Capacitor Model}

UC is an electrochemical capacitor that has high capacitance at a small voltage near $2.5 \mathrm{~V}$. In addition, UC has an energy density of 100-times and a power density about 10-times of the conventional BESS. Moreover, it has low series resistance and free from maintenance with long lifetime [29]. Furthermore, it has the ability to absorb energy in cas of excess generation and release a large amount of power at a very short time duration at the peak-load interval. Neglecting non-linearities, the transfer function of UC can be presented as follows [15]:

$$
G_{U C}(s)=\frac{K_{U C}}{1+s T_{U C}}
$$

where $K_{U C}$ and $T_{U C}$ are the gain and time constant of $U C$, respectively.

High-order mathematical models for WTG, STPG, PV, DEG, FW, AE, FC and EV with non-linearities should be employed for the accurate and practical simulation of their dynamic performance. However, for large systems, simplified models can be utilized to get the performance for a certain scenario [14]. Hence, the system performance with applying the intended control scheme is checked under different scenarios to verify its effectiveness and robustness to show the superiority points of the control approach so as to overcome this problem and solve the main deficit of the previous researches. That is why simplified transfer functions are used to represent all components of the system as indicated in Figure 2.

\subsection{Electric Vehicle Model}

In Japan, the driving distances of about $50 \%$ of the cars per one day is less than $30 \mathrm{~km} \mathrm{[16].}$ This action is still predicted in future with a high penetration of EVs. Actually, every EV faces difficulty to go on a long trip because its driving distance per one charge is shorter that that of gasoline vehicles for one fill. However, EV can be easily charged at many places such as office parks, garages or even at supermarkets. As a result, it is expected that the EV users are intending to charge it repeatedly [49]. Once EV is plugged in, its battery state of charge (SOC) will reach full capacity within only a few hours. Therefore, the V2G control approach can be achieved in this paper by controlling the storing/releasing of the EV battery according to the load frequency control (LFC) signal that will be sent from the proposed controller. To achieve the EV consumers' convenience, only EVs that have already finished charging up to $85 \%$ of SOC are controlled. EV is controlled in the range of $85 \pm 5 \%$ of SOC. The lower limit of $80 \%$ is taken into account such that the EV user would like to have high SOC at some level to be ready at any time for the next trip. In addition, the upper limit of $90 \%$ is decided because the lifetime of the battery becomes shorter when SOC reaches near $100 \%$. All EVs considered in this paper are passenger cars. The equivalent EV model applied in this research is presented in Figure 3 [33]. In this figure, $u$ is the controller output signal; $T_{E V}$ is the time constant of $\mathrm{EV}$; and $\pm \alpha_{e}$ are the inverter capacity limits. Furthermore, the symbols $\pm \sigma_{e}, E_{\max }$ and $E_{\min }$ refer to the power ramp rate limits, maximum and minimum controllable energies of the EV battery, respectively. When $P_{E V}>0$, it means that the EV is in the charging mode, and for $P_{E V}<0$, the EV is in the discharging mode. 


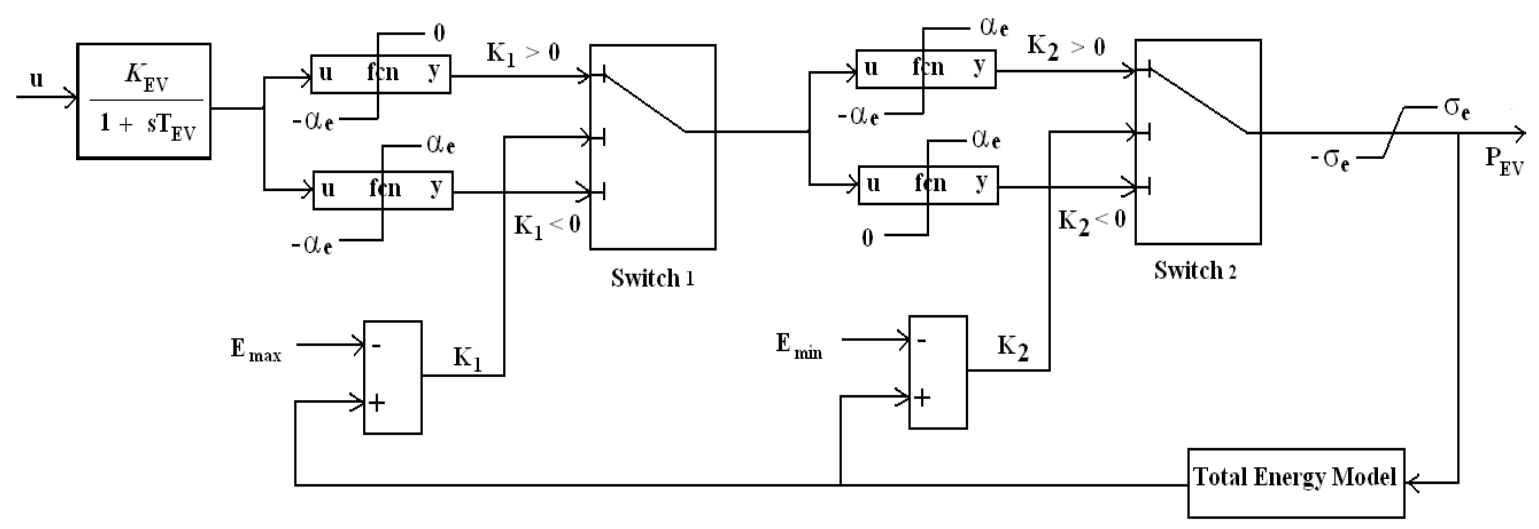

Figure 3. The equivalent EV model.

\subsection{Power and Frequency Deviations}

We must control total power generated to meet demand so as to guarantee an effective performance of the system as output powers of WTG, STPG and PV vary with time. This is can be done by controlling the supply error $\Delta P_{e}$, which is the total power generation minus $P_{L}$, and can be expressed as follows:

$$
\Delta P_{e}=\left(P_{W T G}+P_{S T}+P_{P V}+P_{d}+P_{F C} \pm P_{U C} \pm P_{F W} \pm P_{E V}-P_{L}\right)
$$

The system frequency fluctuates depending on the generated power fluctuations. So, The frequency deviation of the power system $\Delta F$ can be discussed as:

$$
\Delta F=\frac{\Delta P_{e}}{K_{f}}
$$

where $K_{f}$ is system frequency characteristic constant for hybrid power system. This expression is valid only for ideal conditions. But in the practical implementation, there will be a time delay in the frequency characteristics. Hence, the model of frequency deviations is adjusted as:

$$
\Delta F=\frac{\Delta P_{e}}{K_{f}(1+s T)}=\frac{\Delta P_{e}}{s M+D}
$$

where $T, M$ and $D$ are the frequency characteristic time constant, inertia constant and load damping constant, respectively [50].

\section{Controllers Design Approach}

\subsection{Minimal-Order Observer Implementation}

For improving the frequency control performance of the hybrid system that includes renewable sources such as WTG and PV, the estimation for $\Delta P_{e}$ is a vital issue to face some problems such as the fact that some states can not be measured in the system and also model uncertainties issues. One advantage for the proposed observer compared to a full-order one is the fact that it is of minimum-order and subsequently has less parameters. In addition, the intended minimal-order observer can estimate the required signal successfully even with only arbitrarily-given observer poles. Moreover, the high-order observer is treated to be a major deficit to state-space theory for years, as it limits the practical implementation. So, the minimal-order observer is usually desired for the practical 
use and easy implementation [51]. $P_{d}$ and $P_{C}$ are estimated using $\Delta F$ and the input signal of governor $P_{1}$, that is got from $\Delta F$. The state-space equations can be expressed as below:

$$
\begin{gathered}
X^{\cdot}=A X+B P_{1} \\
Y=C X
\end{gathered}
$$

where,

$$
\begin{gathered}
A=\left[\begin{array}{cccc}
-D / M & 1 / M & 0 & 1 / M \\
0 & -1 / T d & 1 / T d & 0 \\
0 & 0 & -1 / T g & 0 \\
0 & 0 & 0 & 0
\end{array}\right] \\
B=\left[\begin{array}{c}
0 \\
0 \\
1 / T g \\
0
\end{array}\right] \\
C=\left[\begin{array}{cccc}
1 & 0 & 0 & 0
\end{array}\right]
\end{gathered}
$$

where $T_{g}$ and $T_{d}$ are the governor and diesel generator time constants, respectively. Four state variables are discussed represent $\Delta F, P_{d}$, output power of governor and $P_{C}$, respectively. $\mathrm{Y}$ is $\Delta F$ only. The actual values of $M, D, T_{g}$ and $T_{D}$ are $0.2 \mathrm{puMW} / \mathrm{Hz}, 0.012 \mathrm{puMW} / \mathrm{Hz}, 0.1 \mathrm{~s}$ and $2 \mathrm{~s}$, respectively. The basic structure of the minimal-order observer is presented in Figure 4, that can be derived in the following steps [52]:

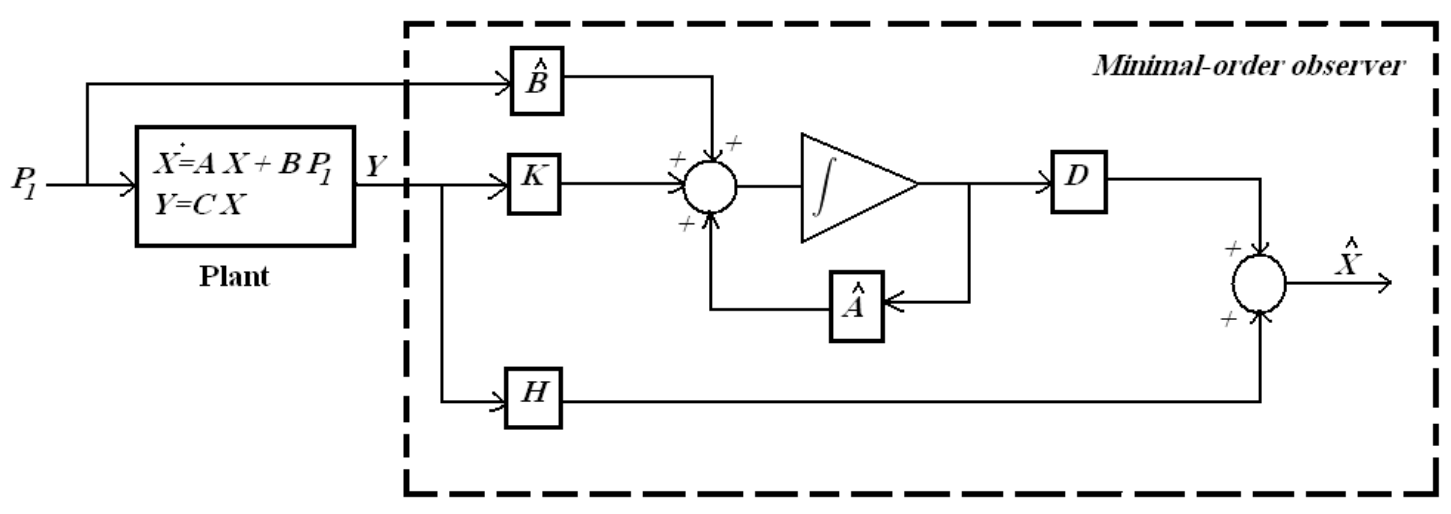

Figure 4. Minimal-order observer.

- Step 1. A matrix $S$ is formulated in the form:

$$
S=\left[\begin{array}{c}
C \\
W
\end{array}\right]=\left[\begin{array}{llll}
1 & 0 & 0 & 0 \\
0 & 1 & 0 & 0 \\
0 & 0 & 1 & 0 \\
0 & 0 & 0 & 1
\end{array}\right]
$$

where $W$ is a suitable matrix for $|S| \neq 0$. 
- Step 2. Calculate the following equations:

$$
\begin{gathered}
S A S^{-1}=\left[\begin{array}{c|lcc}
-0.06 & 5 & 0 & 5 \\
0 & -0.5 & 0.5 & 0 \\
0 & 0 & -10 & 0 \\
0 & 0 & 0 & 0
\end{array}\right]=\left[\begin{array}{lll}
A_{11} & A_{12} \\
A_{21} & A_{22}
\end{array}\right] \\
\left.S B=\left[\begin{array}{c}
0 \\
0 \\
10 \\
0
\end{array}\right]=\frac{\left[B_{1}\right.}{B_{2}}\right]
\end{gathered}
$$

- $\quad$ Step 3. Derive $\hat{A}$ and $\hat{B}$ using $L$ with selected poles

$$
\begin{gathered}
\hat{A}=A_{22}-L A_{12}=\left[\begin{array}{ccc}
13.8938 & 0.5 & 14.3938 \\
-44.1687 & -10 & -44.1687 \\
24.4688 & 0 & 24.4688
\end{array}\right] \\
\hat{B}=-L B_{1}+B_{2}=\left[\begin{array}{c}
0 \\
10 \\
0
\end{array}\right] \\
L=\left[\begin{array}{l}
L_{1} \\
L_{2} \\
L_{3}
\end{array}\right]=\left[\begin{array}{c}
-2.8788 \\
8.8337 \\
-4.8937
\end{array}\right]
\end{gathered}
$$

where $L$ is calculated by solving the following equation:

$$
|s I-\hat{A}|=\left(s-\gamma_{1}\right)\left(s-\gamma_{2}\right)\left(s-\gamma_{3}\right)
$$

The poles of the minimal-order observer are chosen as: $\gamma_{1}=-0.45, \gamma_{2}=-12.5, \gamma_{3}=-17.4$ by good estimation to achieve the proposed simulation results.

- Step 4. Use the above values to get the observer parameters as follows:

$$
\begin{gathered}
K=\hat{A}+A_{21}-L A_{11}=\left[\begin{array}{c}
-106.1919 \\
255.4941 \\
190.4770
\end{array}\right] \\
D=S\left[\begin{array}{l}
0 \\
L
\end{array}\right]=\left[\begin{array}{lll}
0 & 0 & 0 \\
1 & 0 & 0 \\
0 & 1 & 0 \\
0 & 0 & 1
\end{array}\right] \\
H=S\left[\begin{array}{l}
I \\
L
\end{array}\right]=\left[\begin{array}{cc}
1 \\
-2.8788 \\
8.8337 \\
-4.8937
\end{array}\right]
\end{gathered}
$$

Then, the proposed minimal-order observer is constructed using these parameters. Hence, the estimated supply error $\Delta \hat{P}_{e}$ is calculated using the estimated values of output power of DEG, $\hat{P}_{d}$, and combined power, $\hat{P}_{C}$, by the following equation:

$$
\Delta \hat{P}_{e}=\hat{P}_{d}+\hat{P}_{C}
$$




\subsection{Polar Fuzzy-Based Controllers Scheme}

The performance of FLC is limited already because of its large rules number which lead to large computation time and memory requirement too. Therefore, this problem is solved by utilizing PF. $\mathrm{PF}$ is helpful in cases which get polar coordinates bases or in situations in which the cyclic values for variables exist. PF differs from conventional fuzzy resonance only for polar fuzzy sets, that can be defined in terms of angle and, then, repeat their shapes every $2 \pi$ radians [53].

In this section, two PF controllers are formulated, as shown in Figure 5. ACE is calculated as the sum of $e \Delta P_{e}$ and $e \Delta F$ multiplied by a fixed coefficient $\mathrm{K}$; where $e \Delta P_{e}$ is the difference between estimated supply error $\Delta \hat{P}_{e}$ and supply error command value $\Delta P_{e}^{*}$, which has zero value. $e \Delta F$ is difference between frequency deviation $\Delta F$ and frequency deviation command value $\Delta F^{*}$, which has zero value. $e \Delta F$ is multiplied by factor $\mathrm{K}$ because $\Delta \hat{P}_{e}$ is very large compared to $\Delta F$. Trial and error criteria is used to get the value of $\mathrm{K}$ such that $\triangle F$ is small enough. Then, $\mathrm{ACE}$ is considered in the frequency domain. The two inputs of the first controller are the low-frequency component of ACE and its variation with time, respectively. Controller output is then fed as the control signal to modify output powers of DEG, FC and stored/released power of EV, respectively. Moreover, high-frequency component of ACE and its change with time are two inputs for the second controller. The output signal of controller is utilized to modify the stored and released powers of UC and FW. Time delays are used to get the signals of the low-frequency component of ACE variation with time $\left(\triangle A C E_{-}\right.$low) and the change of high-frequency component of ACE with time $\left(\triangle A C E_{-}\right.$high). The delay sample period is chosen to be $0.0044 \mathrm{~s}$ for the best performance of the intended control approach. The time constants of low-pass filter (LPF) and the high-pass filter (HPF) are $20 \mathrm{~s}$ and $10 \mathrm{~s}$, respectively. These values have been specified by sensitive estimation to indicate the frequency range of each control variable so as to cover the whole frequency space of ACE.

The basic structure of PF controller is presented in Figure 6. In PF, no need to utilize two input gains for $\mathrm{ACE}$ and its variation with time $\triangle \mathrm{ACE}$, because the polar angle $(\theta)$, as only one input which depends on these input ratio is used. Therefore, only one gain $K_{a c c}$ is utilized. $K_{a c c}$ is used to choose the variable that has more weight in the magnitude $(M a g)$. For the intended control scheme, $\triangle \mathrm{ACE}$ is chosen to have more weight on Mag with $K_{a c c}=1.35$. ACE and the product of $\left(\triangle A C E \times K_{a c c}\right)$ are defined in the complex plan, and then, the complex quantity is converted to equivalent polar coordinates (angle and magnitude). Then, $\theta$ only is used as the input to FLC. Therefore, the output of the FLC unit is a function of angle $(\theta)$, i.e., $U_{F L C}=f 1(\theta)$, and the final PF controller output $u$ is calculated in the next equation $[34,53,54]$ :

$$
u=U_{F L C} \times M a g
$$

where,

$$
\begin{gathered}
M a g=\sqrt{\left(\triangle A C E \times K_{a c c}\right)^{2}+(A C E)^{2}} \\
\theta=\tan ^{-1}\left(\left(\triangle A C E \times K_{a c c}\right) / A C E\right)
\end{gathered}
$$




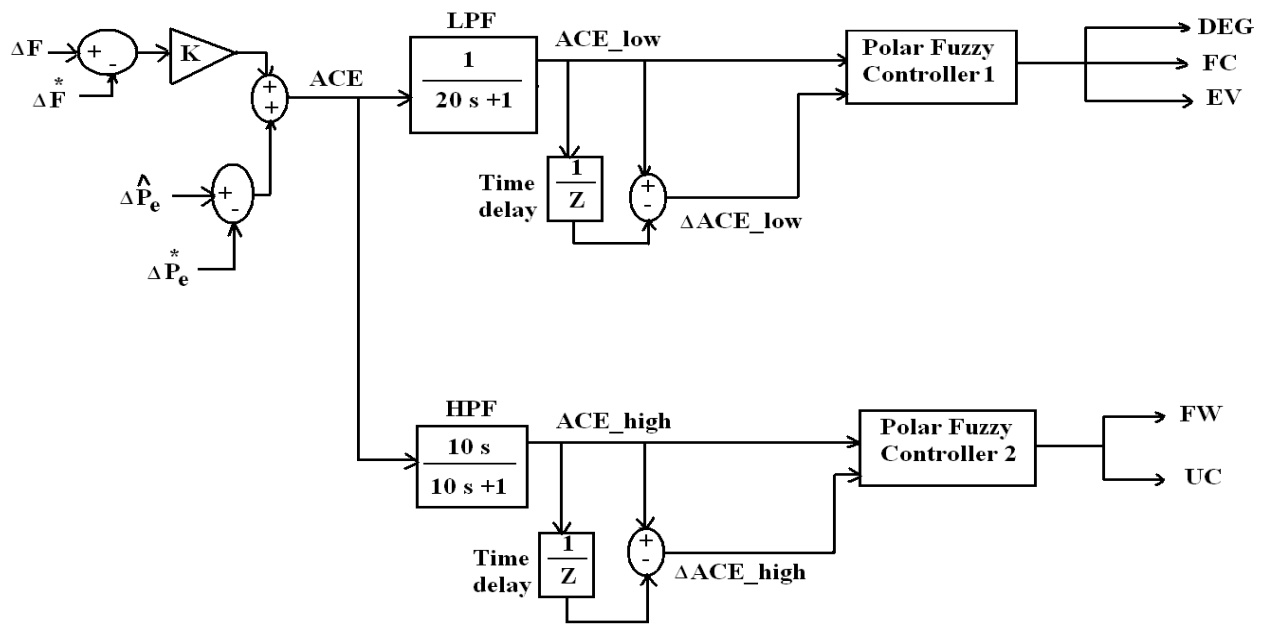

Figure 5. Polar fuzzy (PF) controllers' scheme.

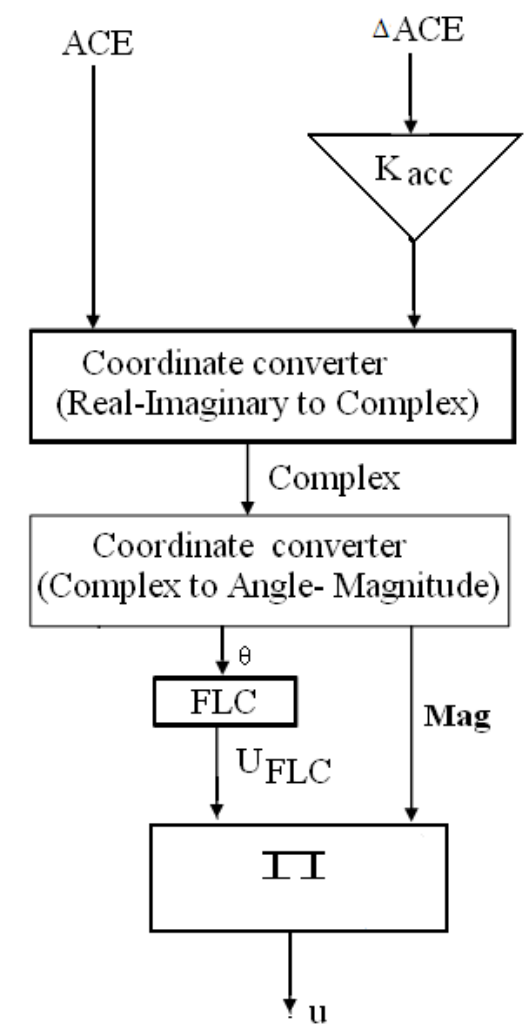

Figure 6. PF controller model.

The proposed PF control scheme is discussed in Figure 7, which can be summarized as follows:

- In Section I, the control signal from FLC, $U_{F L C}$, should be large negative, as both scaled ACE and $\triangle \mathrm{ACE}$ are positive.

- In Section II, the control signal from FLC would be medium negative as scaled ACE is large positive and scaled $\triangle \mathrm{ACE}$ is small negative.

- In Section III, the control signal from FLC should be small negative as scaled ACE is small negative and scaled $\triangle \mathrm{ACE}$ is large positive.

- In Section IV, the control signal from FLC should be large positive, as both scaled ACE and $\triangle \mathrm{ACE}$ are negative. 
- In Section $\mathrm{V}$, the control signal from FLC should be medium positive as scaled ACE is large negative, while scaled $\triangle \mathrm{ACE}$ is small positive.

- In Section VI, the control signal from FLC should be small positive, as scaled ACE is small positive and scaled $\triangle \mathrm{ACE}$ is large negative.

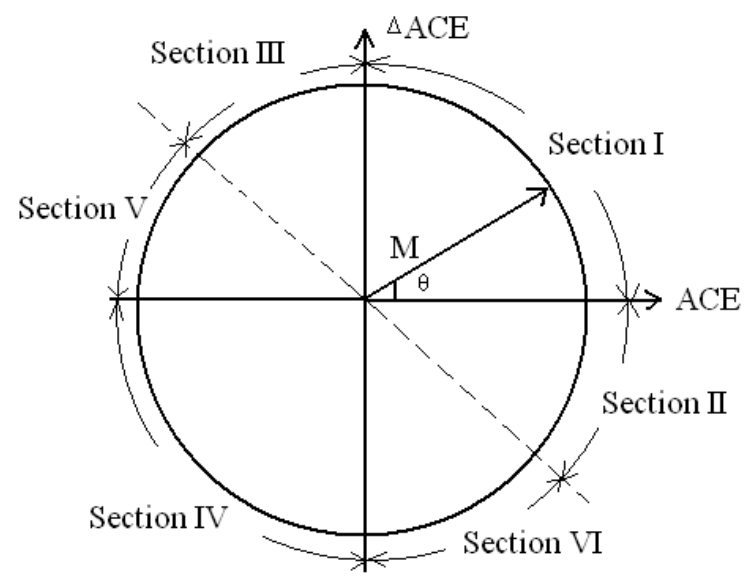

Figure 7. PF phase plan.

Fuzzy membership functions for input and output patterns of intended control approach are addressed in Figures 8 and 9, respectively. For each controller, two fuzzy sigmoid functions are utilized, that are large positive $(L P)$ and large negative $(L N)$ for $\theta$ as input. These two membership functions are complementary to each other and are defined in the range of $45-405^{\circ}$. The controller output $U_{F L C}$ is expressed as two variables, positive $(P)$ and negative $(N)$, that are presented as triangular membership functions. As the FLC of PF is a single input single output (SISO) system, therefore two simple rules can only be sufficient in the rule base:

$$
\begin{aligned}
& \text { If } \theta \text { is LP Then } U_{F L C} \text { is } N \\
& \text { If } \theta \text { is LN Then } U_{F L C} \text { is } P
\end{aligned}
$$

At $\theta$ equal to $45^{\circ}$ or $405^{\circ}$, the value of membership function $L P$ is maximum, but for $L N$ is minimum, so that $U_{F L C}$ is maximum negative. At angles of $135^{\circ}$ or $315^{\circ}$, the value of membership function of $L P$ and also $L N$ is the same, that is why $U_{F L C}$ is minimum (zero). On the other hand, at angle of $225^{\circ}$, the value of the membership function $L P$ is minimum and for $L N$ is maximum, so $U_{F L C}$ is maximum positive. The flowchart for complete design process of the proposed PF scheme is presented in Figure 10.

The plots of the singular values for control loops with/without the intended scheme are presented in Figures 11 and 12. It is evident from Figure 11 that the singular values plot of the frequency control loop $\left(\Delta F^{*} \rightarrow \Delta F\right)$ holds a large value of gain in the low-frequency domain without using proposed technique. Therefore, frequency fluctuations of power system will grow in the this domain. In addition, the singular value has a resonance point as indicated in this figure. The frequency associated to the resonance point can be easily calculated, and the value is $12.7 \mathrm{rad} / \mathrm{s}$. The resonance point might affect frequency control of system. On the other hand, the singular values plot of the supply error control loop $\left(\Delta P_{e}^{*} \rightarrow \Delta P_{e}\right)$ has an integral characteristic at the low frequency domain without utilizing intended approach as pointed at Figure 12. Due to the integral characteristic, the supply error continues growing so the characteristic may be eliminated. 


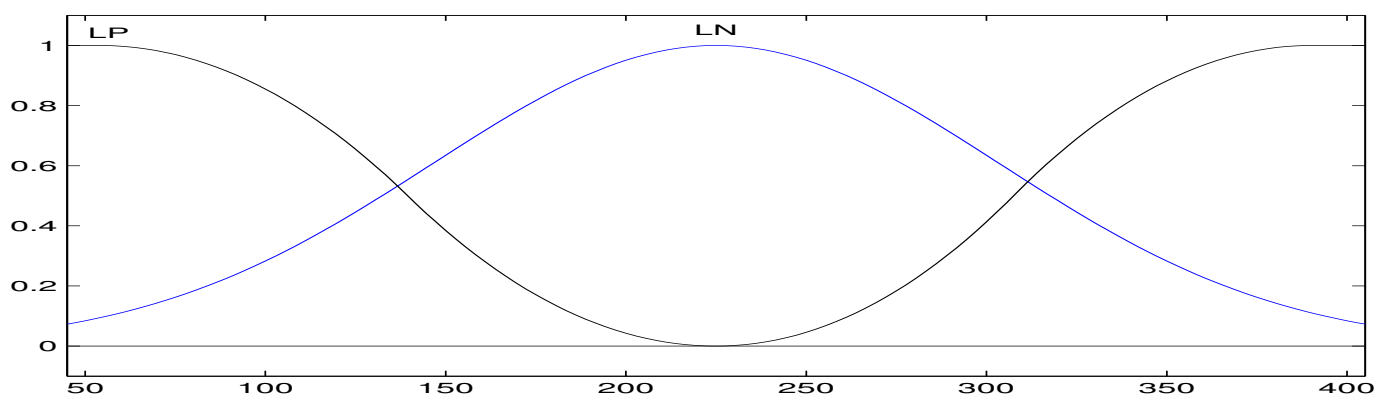

(a)

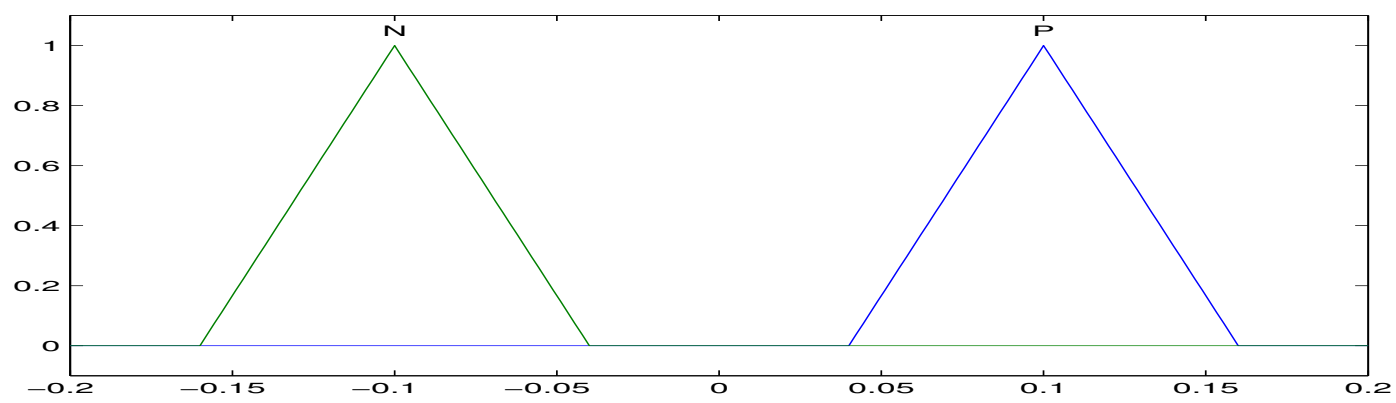

(b)

Figure 8. (a) Input membership functions for first PF controller; (b) output membership functions for first PF controller.

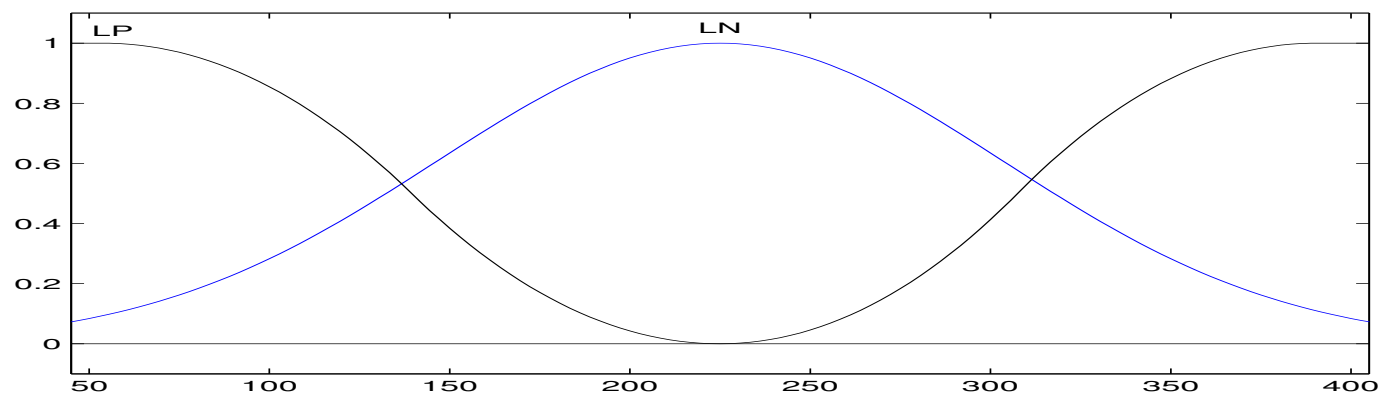

(a)

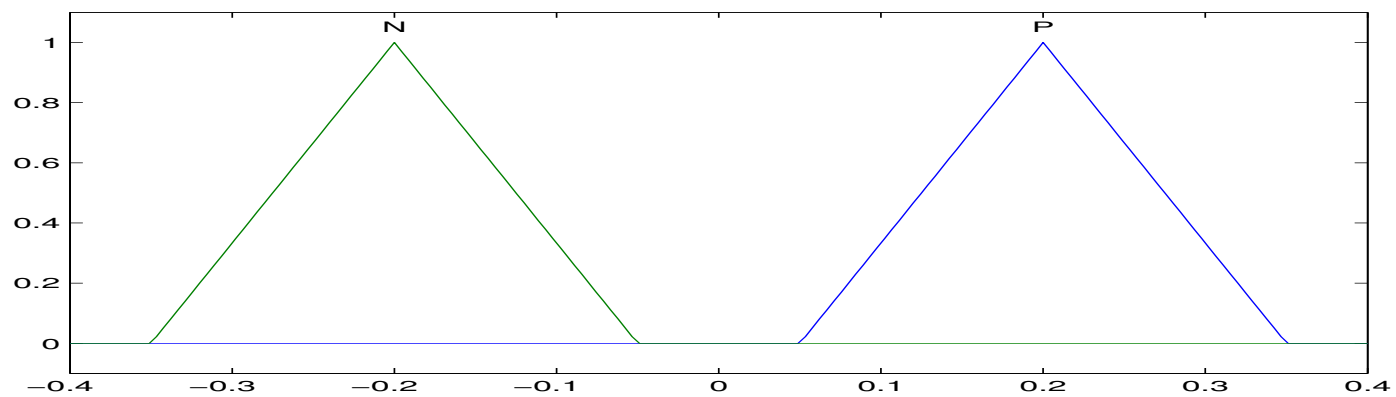

(b)

Figure 9. (a) Input membership functions for the second PF controller; (b) output membership functions for second PF controller. 


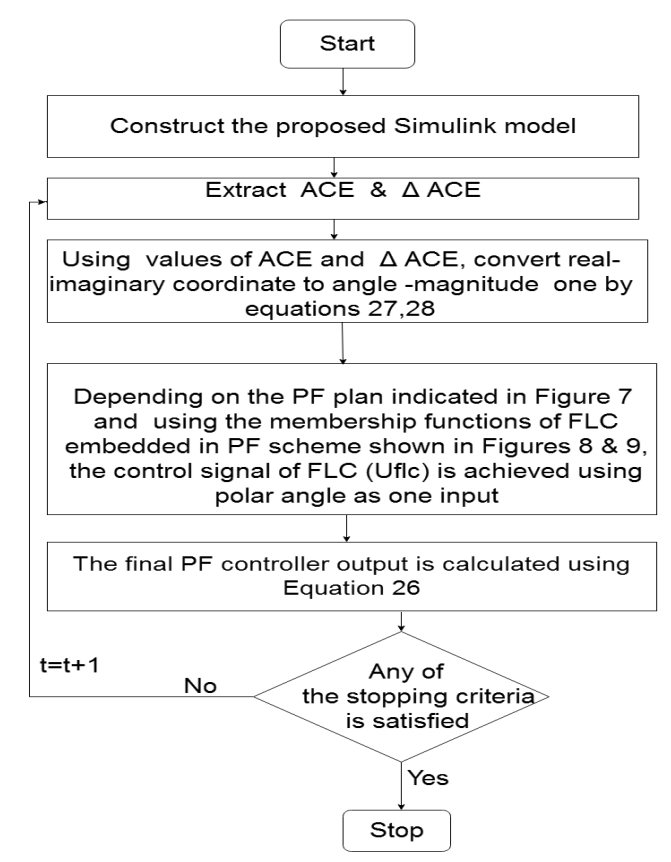

Figure 10. Flowchart of the PF scheme design process.

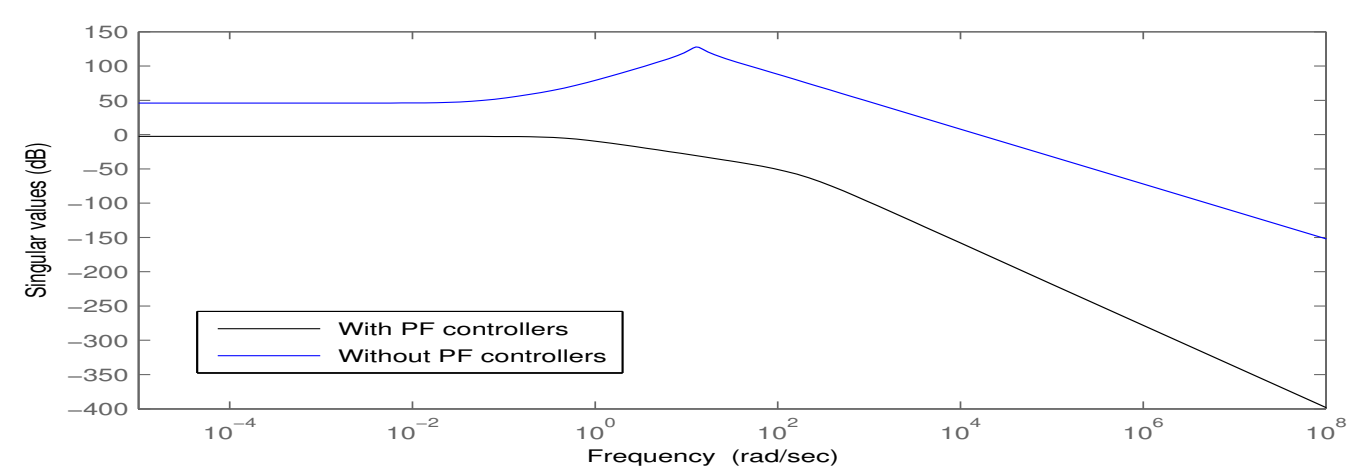

Figure 11. Singular values plot of frequency control loop $\left(\Delta F^{*} \rightarrow \Delta F\right)$.

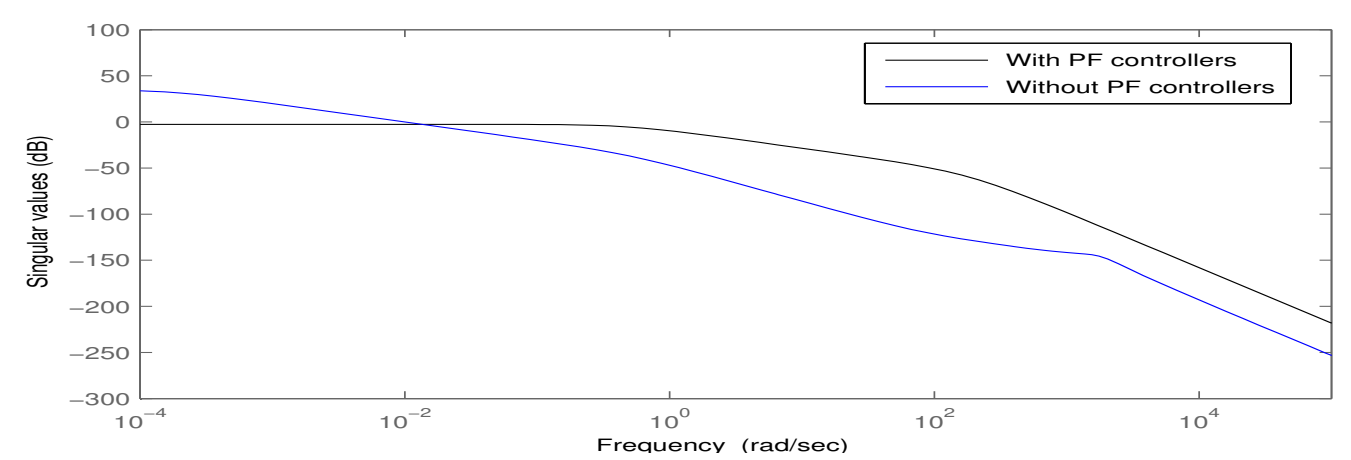

Figure 12. Singular values plot of supply error control loop $\left(\Delta P_{e}^{*} \rightarrow \Delta P_{e}\right)$.

\section{Results}

Some simulations of the system for different scenarios have been held in this part using the Simulink environment of MATLAB software to testify robustness for intended technique. The simulations are performed for 1800 seconds interval. The hybrid system parameters utilized 
for simulations are presented in Table $1[33,50]$. The proposed PF scheme is compared with the conventional FLC for various sets of power generations and energy storage systems to validate effectiveness for intended technique. In FLC, two controllers are implemented. The role of each one is to suppress one frequency component of ACE the same as the intended PF control scheme. One frequency component of $\mathrm{ACE}$ and its variation with time are used as two inputs for every FLC approach, while its output control signal is fed to system elements to control frequency components of ACE. Fuzzy sets with their associated membership functions of FLC are then presented in Figures 13 and 14, respectively, while their control rules are expressed in Table 2. The membership functions of each FLC approach for inputs and outputs are set as negative large (NL), negative medium 1 (NM1), negative medium 2 (NM2), negative small (NS), zero (Z), positive small (PS), positive medium 1 (PM1), positive medium 2 (PM2) and positive large (PL).

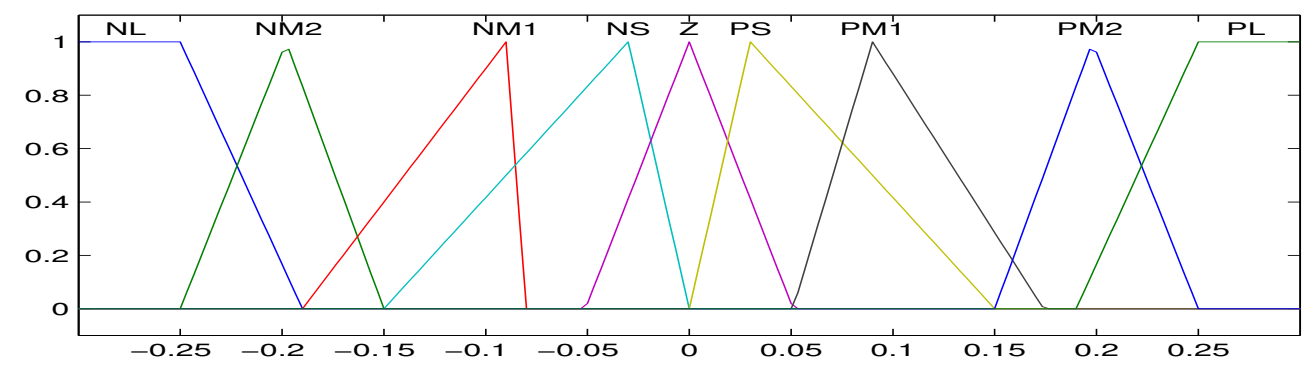

(a)

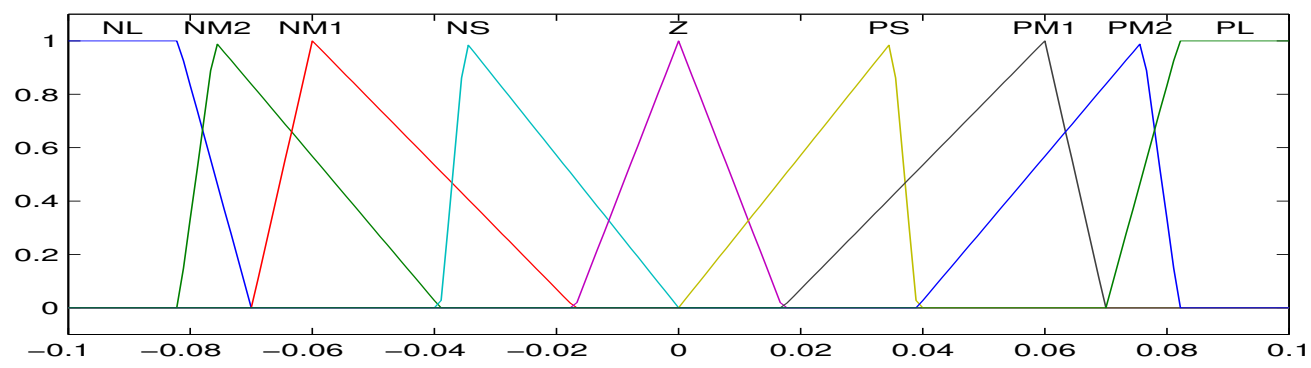

(b)

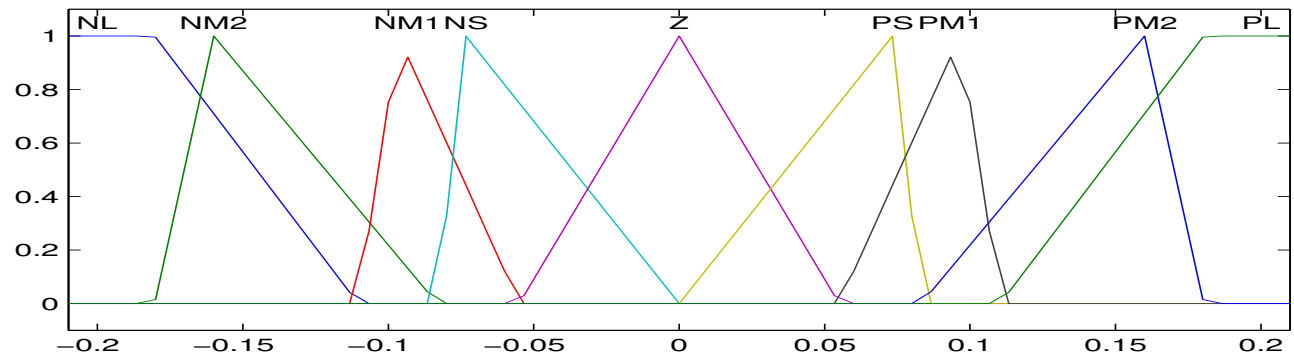

(c)

Figure 13. (a) First input membership functions for the first fuzzy logic control (FLC) scheme; (b) second input membership functions for the first FLC scheme; (c) output membership functions for the first FLC scheme. 


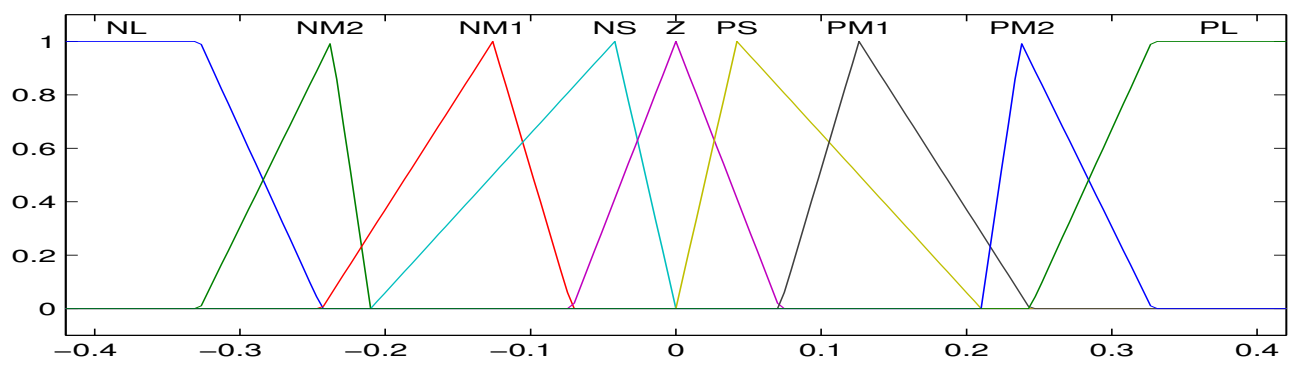

(a)

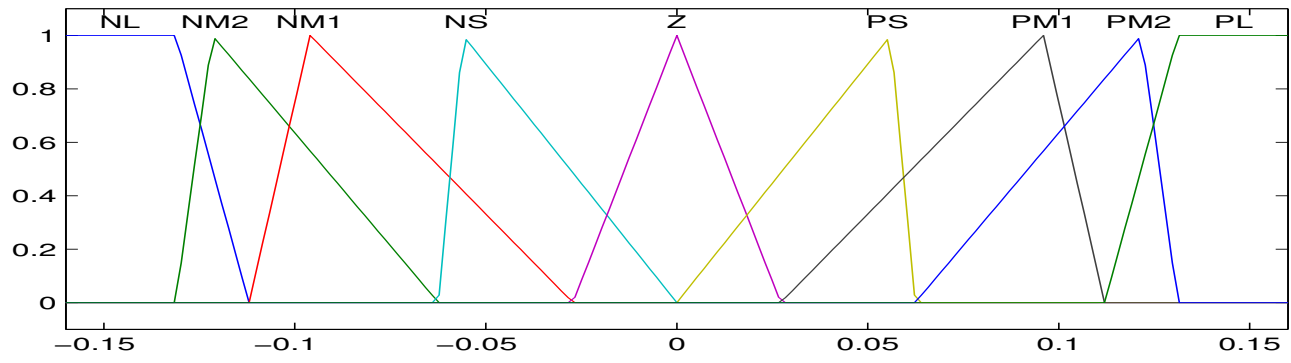

(b)

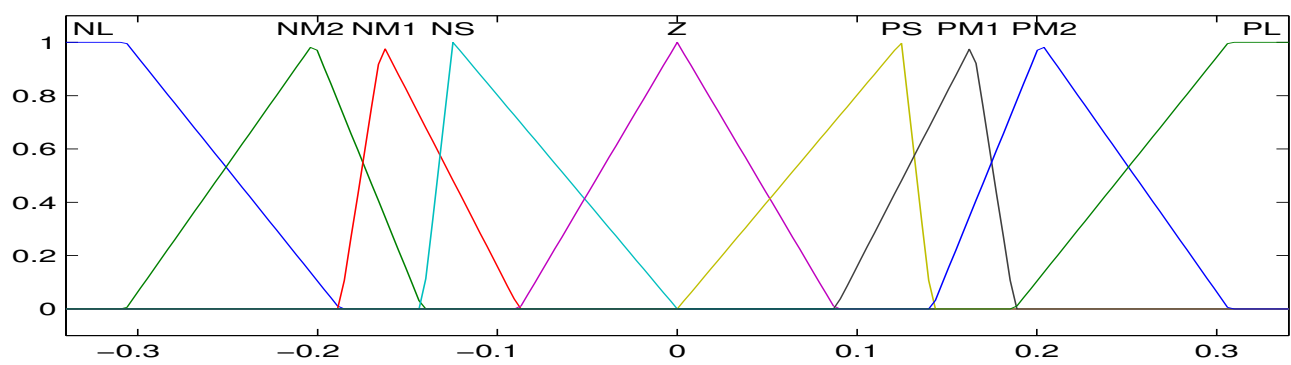

(c)

Figure 14. (a) First input membership functions for the second FLC scheme; (b) second input membership functions for the second FLC scheme; (c) output membership functions for for the second FLC scheme.

Table 1. Hybrid power system parameters.

$$
\begin{aligned}
& \beta=0, \text { speed regulation } R=2.4 \mathrm{~Hz} / \text { puMW and } K=500 \\
& K_{W T G}=1 \text { and } T_{W T G}=1.5 \mathrm{~s} \\
& K_{U C}=1 / 100 \text { and } T_{U C}=0.15 \mathrm{~s} \\
& \alpha_{e}=0.36 \text { puMW and } \sigma_{e}=0.075 \mathrm{puMW} / \mathrm{s} \\
& K_{S}=1.8, K_{T}=1, T_{S}=1.8 \mathrm{~s} \text { and } T_{T}=0.3 \mathrm{~s} \\
& K_{P V}=1, T_{P V}=1.85 \mathrm{~s}, T_{E V}=1 \mathrm{~s} \text { and } K_{E V}=1 / 100 \\
& K_{A E}=1 / 25, T_{A E}=0.5 \mathrm{~s}, K_{F C}=1 / 5 \text { and } T_{F C}=4 \mathrm{~s} \\
& K_{F W}=1 / 300 \text { and } T_{F W}=0.1 \mathrm{~s}
\end{aligned}
$$


Table 2. Fuzzy control rules. NL, negative large; NM, negative medium; NS, negative small; Z, zero; PS, positive small; PM, positive medium; PL, positive large.

\begin{tabular}{cccccccccc}
\hline ACE & NACE & NM2 & NM1 & NS & Z & PS & PM1 & PM2 & PL \\
\hline NL & PL & PL & PM2 & PM2 & PM1 & PM1 & PS & PS & Z \\
NM2 & PL & PM2 & PM2 & PM1 & PM1 & PS & Z & Z & Z \\
NM1 & PL & PM2 & PM1 & PM1 & PS & Z & Z & Z & Z \\
NS & PL & PM2 & PM1 & PS & PS & Z & Z & Z & NS \\
Z & PM2 & PM1 & PS & PS & Z & NS & NM1 & NM1 & NM2 \\
PS & PS & Z & Z & Z & NS & NM1 & NM1 & NM2 & NM2 \\
PM1 & Z & Z & Z & Z & NS & NM1 & NM2 & NM2 & NL \\
PM2 & Z & Z & Z & NS & NM1 & NM2 & NM2 & NL & NL \\
PL & Z & NS & NM1 & NM2 & NM2 & NM2 & NL & NL & NL \\
\hline
\end{tabular}

\subsection{Case 1}

This scenario has been dealt as fundamental case that contains all of the power generations with energy storage systems for hybrid system. The robustness for proposed technique is checked under a wide range of scenarios as the sudden increase/decrease of the wind speed $V_{W}$, load demand, solar radiation $\phi$ and the linear increase in $\phi$. The associated time-domain results are indicated in Figure 15, and their corresponding response is analyzed in the next part.
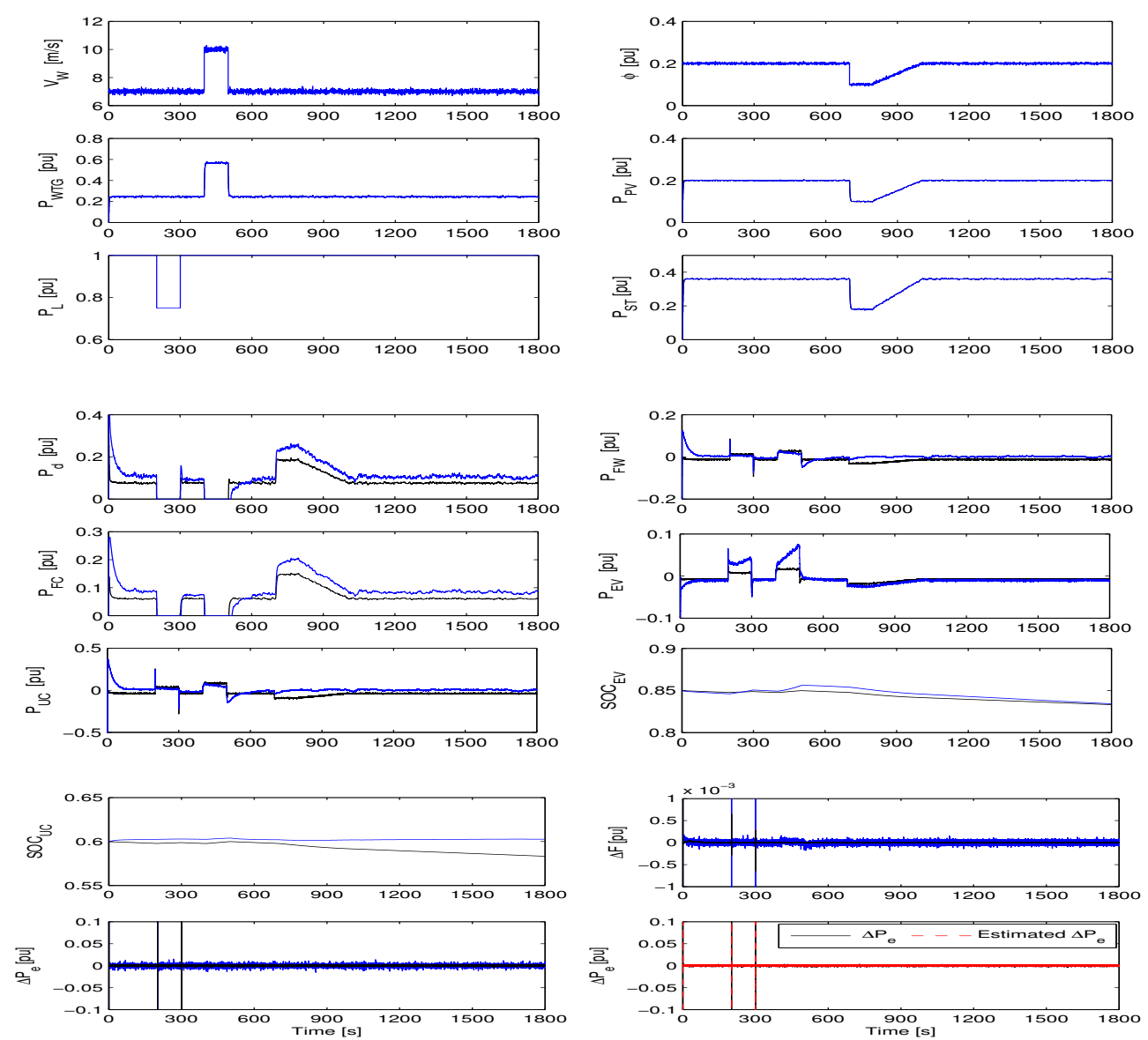

Figure 15. Case 1 simulation results: PF (black), FLC (blue).

It is clear from this figure that DEG and FC with FLC have a high value of power with large oscillations and huge overshoot reaching up to $0.3 \mathrm{pu}$ for DEG and $0.2 \mathrm{pu}$ for FC compared to 
the proposed PF scheme, which succeeded to damp the deviations of $P_{D E G}$ and $P_{F C}$ significantly. In addition, the proposed control approach can manage the charged/released power of FW and UC depending on the variations of wind speed, load demand and solar radiation with less fluctuations and overshoots compared to the conventional FLC in which FW and UC cannot respond to the majority of the sudden changes. Moreover, high fluctuations seem significant for the charged/discharged power of EV using FLC in comparison with the intended PF technique that has the capability to mitigate the oscillations of $P_{E V}$ dramatically, as shown in Figure 15. Furthermore, the customers' comfort is taken into account, and the SOC of the EV battery is within the specified limits for both control schemes. In addition, the proposed PF technique and FLC succeeded at keeping the SOC of UC within its safe limits (25-90\%) as mentioned in [55] with using $60 \%$ as the initial value for SOC. Also, Figure 15 confirms obviously capability for intended PF approach to minimize fluctuations of $\Delta F$ and $\Delta P_{e}$ in a great manner compared to conventional FLC. Moreover, the minimal-order observer succeeded to estimate $\Delta P_{e}$ accurately as indicated in the figure.

\subsection{Case 2}

The hybrid power system in this scenario contains all of the generation sources and energy storage systems, the same as Case 1. The main difference is the fact that the output powers of WTG, PV and STPG are summed, and only $50 \%$ of it is fed to the power system, while the remaining percentage is utilized by AE to produce hydrogen, which will be used later as fuel for FC. Furthermore, a balanced fault at one of the wind turbine generators is considered in Case 2 at time $t=1000 \mathrm{~s}$, which leads to the outage of this unit. The fault is cleared at $t=1100 \mathrm{~s}$. Figure 16 discusses the simulation results of this case study, and their associated response can be examined in the next part.

The proposed PF scheme can suppress the deviations of $P_{D E G}$ with less overshoot compared to conventional FLC that has higher fluctuations for the output power of DEG with larger overshoot reaching up to $0.5 \mathrm{pu}$. Moreover, intended control scheme still can control the stored/released powers of FW and UC in accordance with the changes in wind speed, load demand and solar radiation so as to keep the supply error and consequently the frequency deviation almost zero at any time; while the traditional FLC cannot drive $P_{F W}$ and $P_{U C}$ to follow the rapid variations of wind speed, load demand, and solar radiation as shown in the figure. In addition, Figure 16 indicates that the proposed PF control technique mitigated the fluctuations of the charged/released power of EV significantly compared to FLC. Furthermore, both control approaches succeeded to hold the SOC of EV battery within the pre-designed limits to ensure the consumers' conveniences and to increase battery lifetime together. Also, SOC of UC is still within the specified limits for both control approaches. In addition, the proposed PF scheme still can face such severe scenario with damping $\Delta P_{e}$ and $\Delta F$ fluctuations dramatically compared to the conventional FLC with an effective estimate for the minimal-order observer as presented in Figure 16.

Generally, the deviations of output power of DEG and charged/discharged powers of FW, UC and EV for both control schemes are less than those in Case 1 thanks to the role of AE to absorb $50 \%$ of WTG, PV and STPG fluctuations. This action improves the power quality of the system significantly. On the other hand, the main deficit for this configuration is due to the need of the hybrid power system for a larger capacity of DEG, FC, AE and hydrogen fuel tank, which will increase the total cost of the system. 

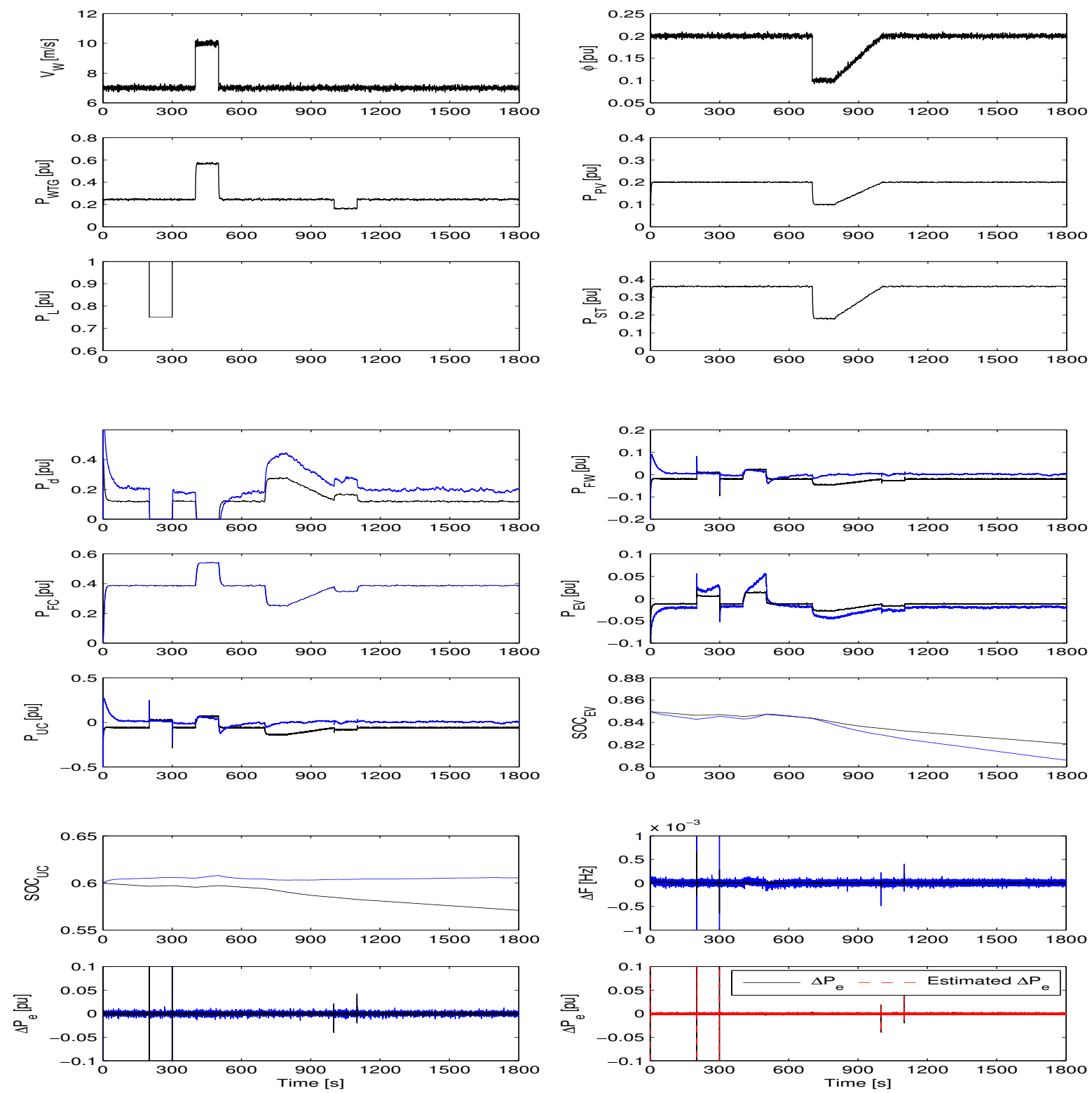

Figure 16. Case 2 simulation results: PF (black), FLC (blue).

\subsection{Case 3}

To testify the effectiveness of the intended control approach, actual wind speed, load demand and solar radiation data of Okinawa, Japan, has been utilized. Wind speed and solar radiation data has been picked up from Japan Weather Association (JWA) [56], while load data are gathered from Okinawa Electric Power Company, Incorporated (OEPC) [57]. Full-day 24-hour data for actual daily wind speed, solar radiation and load demand are utilized in this case. Simulation performances are presented in Figure 17, that will be discussed in the next part. 

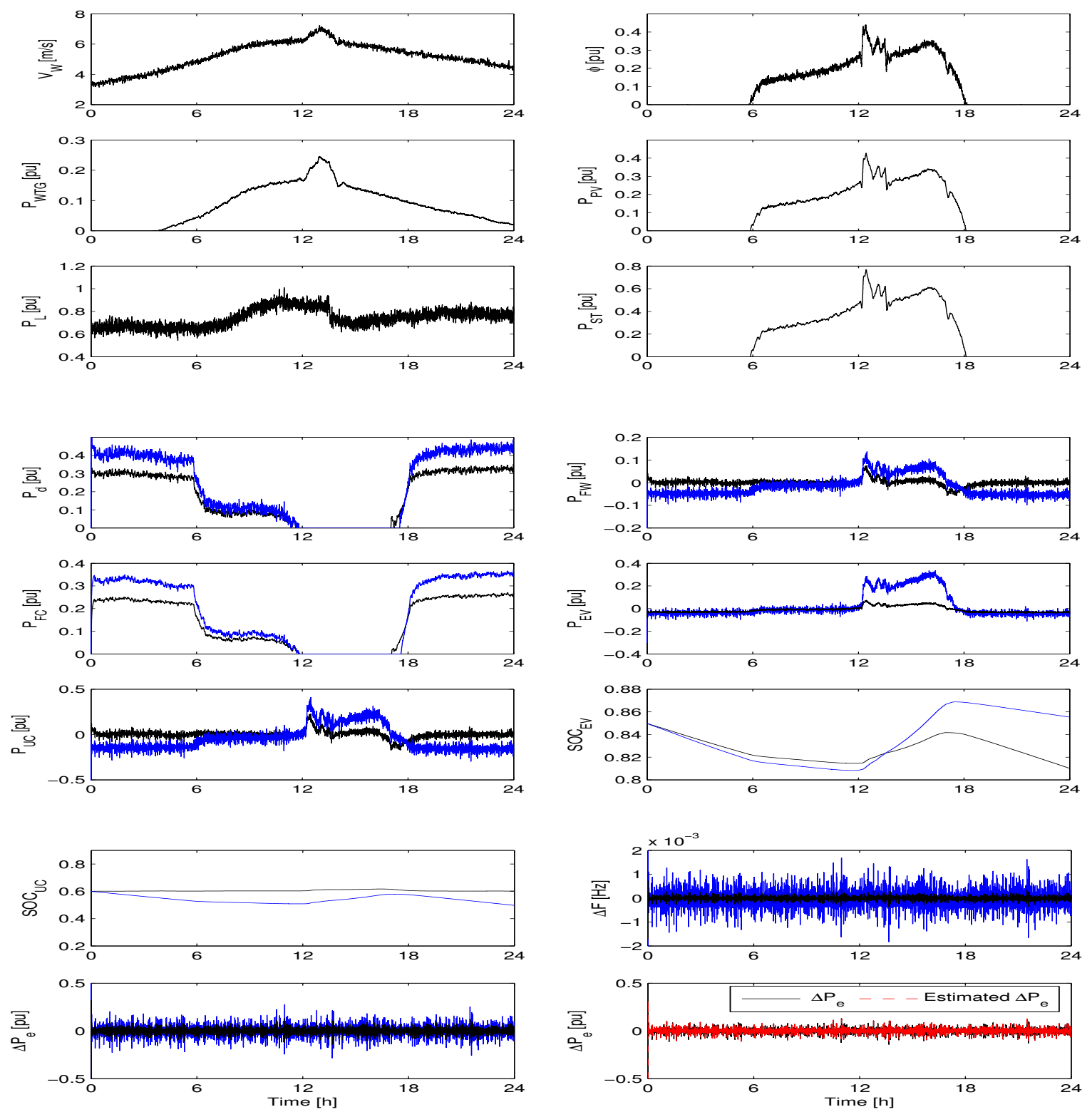

Figure 17. Case 3 simulation results: PF (black), FLC (blue).

The figure clarifies the capability of the intended PF control approach to decrease the fluctuations and overshoots concerning the output of DEG and FC with a clear value compared to the conventional FLC. Moreover, the proposed approach succeeded to mitigate deviations of the stored/released powers of FW and UC dramatically. Furthermore, large fluctuations for $P_{E V}$ appear using traditional FLC reaching to $0.32 \mathrm{pu}$, while the intended $\mathrm{PF}$ technique suppresses the fluctuations of charged/released power of EV, significantly challenging the real variations of wind speed, load demand and solar radiation. In addition, SOC of the EV battery still has the specified limits to guarantee the customers' relief. Moreover, the proposed PF scheme and FLC still have the ability to keep the SOC of UC within its secure limits without any violations. Furthermore, Figure 17 confirms superiority of intended scheme for mitigating fluctuations of $\Delta P_{e}$ and $\Delta F$ dramatically and keeping their values near zero compared to the FLC. Besides that, the minimal-order observer ensures effective achievement for $\Delta P_{e}$ estimation even in such harsh scenario. 
Finally, the suppression effects of the supply error and frequency deviation for the proposed PF control scheme and FLC have been evaluated quantitatively. The evaluation index is the root mean square (RMS) values, which can be expressed in the next equations:

$$
\begin{aligned}
& P_{R M S}=\sqrt{\frac{1}{N} \sum_{i=1}^{N} \Delta P_{e_{i}}^{2}} \\
& F_{R M S}=\sqrt{\frac{1}{N} \sum_{i=1}^{N} \Delta F_{i}^{2}}
\end{aligned}
$$

where $\mathrm{N}, \Delta F_{i}$ and $\Delta P_{e_{i}}$ are the number of samples, frequency deviation and supply error of the sample $i$, respectively. The RMS values of previous three cases are arranged in Table 3 , which clearly indicates that the proposed PF control approach has lower performance index values for both frequency deviation and supply error for all of the previous three cases compared to conventional FLC. These results clarifies significantly ability for intended scheme to achieve desired response for different operating conditions overcoming main drawback concerning majority of the previous studies.

Table 3. RMS values of frequency deviation and supply error for PF and FLC.

\begin{tabular}{llll}
\hline & & PF & FLC \\
\hline \multirow{2}{*}{ Case 1 } & $F_{R M S}$ & $8.1681 \times 10^{-6}$ & $1.2922 \times 10^{-4}$ \\
& $P_{R M S}$ & 0.0057 & 0.0092 \\
\hline \multirow{2}{*}{ Case 2 } & $F_{R M S}$ & $7.6881 \times 10^{-6}$ & $1.2859 \times 10^{-4}$ \\
& $P_{R M S}$ & 0.0052 & 0.0089 \\
\hline \multirow{2}{*}{ Case3 } & $F_{R M S}$ & $1.076 \times 10^{-5}$ & $1.3956 \times 10^{-4}$ \\
& $P_{\text {RMS }}$ & 0.0065 & 0.0156 \\
\hline
\end{tabular}

\subsection{Case 4}

For this scenario, hybrid system is subjected to some parameter variation to investigate its robustness and effectiveness for this issue. The absolute maximum frequency deviation $\left(|\Delta F|_{\max }\right)$ and the absolute maximum supply error $\left(\left|\Delta P_{e}\right|_{\max }\right)$ are checked for the same operating condition of Case 1 , while two of the system parameters are changed from their nominal values. $D$ and $M$ are decreased from $15-75 \%$ of their normal values. Figure 18 demonstrate the values of $|\Delta F|_{\max }$ and $\left|\Delta P_{e}\right|_{\max }$ with $\mathrm{D}$ and $\mathrm{M}$ variations for both of proposed PF control method and conventional FLC. The results clearly indicate that the values of $|\Delta F|_{\max }$ and $\left|\Delta P_{e}\right|_{\max }$ of the proposed PF scheme have very small changes with the system parameter variation compared to those of the traditional FLC. Moreover, the intended PF approach still provides greatly lower $|\Delta F|_{\max }$ and $\left|\Delta P_{\mathcal{e}}\right|_{\max }$ values. Accordingly, the proposed control technique is more robust to the system parameter change compared to FLC. 


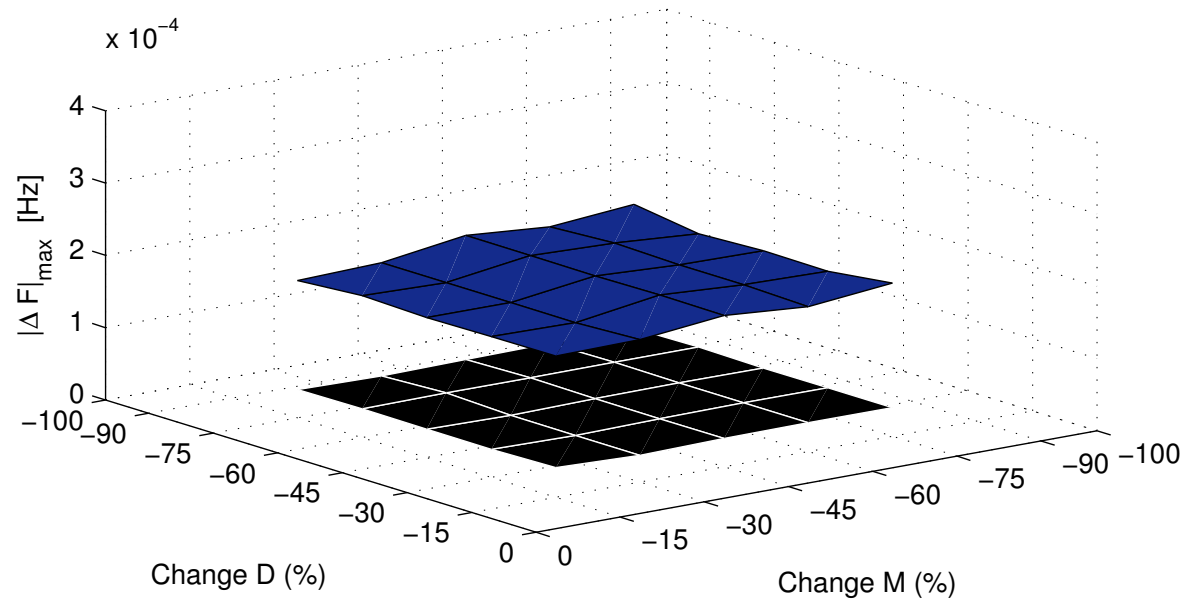

(a)

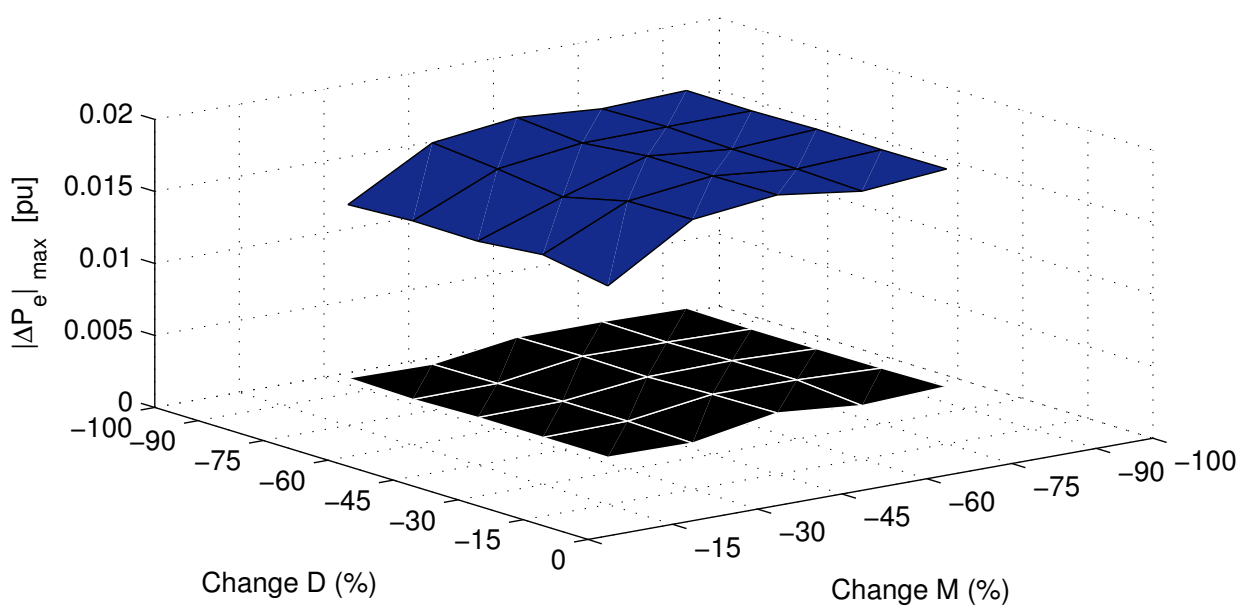

(b)

Figure 18. (a) $|\Delta F|_{\max }$ for system parameters variation: PF (black), FLC (blue); (b) $\left|\Delta P_{e}\right|_{\max }$ for system parameters variation: PF (black), FLC (blue).

\section{Conclusions}

A modern frequency domain PF approach of the hybrid power system has been discussed in this research. The intended system contains the PV array, STPG, DEG, AE, FC, UC, FW and three units of WTG. Furthermore, a new scheme of V2G control is applied to manage the charged/discharged power of the EV battery to overcome the problem of the high cost for the conventional BESS. Moreover, the EV consumers' comfort is taken into consideration via keeping the EV battery SOC within its pre-specified limits. Then, a minimal-order observer is utilized to estimate $\Delta P_{e}$. After that, ACE signal is calculated in terms of $\Delta F$ and $\Delta P_{e}$. ACE is considered in the frequency domain. The PF technique is used to deign two controllers and the duty of each one is to suppress one frequency component of ACE. The high-frequency component of ACE is damped using short time constant elements such as UC and FW. Then, low-frequency component of ACE is minimized by DEG, FC and EV, which have long-time constants. The response of the intended scheme is compared with that of the conventional FLC. Four scenarios are expressed and discussed to validate the effectiveness and robustness of the intended technique for different scenarios. Simulation responses indicate precisely the superiority for intended approach to: 
(1) Manage power flow for $P_{D E G}, P_{F C}, P_{U C}, P_{F W}$ and $P_{E V}$ instantaneously depending on the changes of wind speed, load demand and solar radiation, to mitigate the supply error and system frequency oscillations.

(2) Overcome the major drawback of the conventional FLC represented in the large number of control rules required in formulating its knowledge base that accordingly increase the computational time and memory requirement dramatically. Therefore, the proposed technique used only two control rules for its knowledge base, which makes it beneficial for the practical implementation.

(3) Withstand severe scenarios such as actual data and sudden increase/decrease of wind speed, load demand, solar radiation, faulty conditions in addition to system parameter variations to confirm its robustness and effectiveness for various operating conditions and to compensate the main disadvantage of almost all of the previous researches that can not catch the characteristics of the system for wide range of operating conditions.

(4) Decrease fluctuations of all components of hybrid power system (DEG, FC, UC, FW, EV). Hence, a smaller size of all of these systems will be required if this control scheme is utilized, which modify the system overall efficiency and at the same time decrease its total cost.

Overall, the response of the system is improved greatly with implementing the intended technique. The next duty in the near future will be modifying this research to explore the response of the system, including the detailed nonlinear model of all parts so that the associated research be more practical and solid.

Author Contributions: All of the authors have contributed to this research. Mohammed Elsayed Lotfy, Tomonobu Senjyu and Mohammed. Abdel-Fattah Farahat carried out the study, analyzed the corresponding results and also prepared the manuscript draft. Amal Farouq Abdel-Gawad and Hidehito Matayoshi suggested the study idea and shared in writing and revising the paper. All of the authors revised and approved the manuscript.

Conflicts of Interest: All of the authors declare no conflict of interest.

1. Marzband, M.; Sumper, A.; Gomis-Bellmunt, O.; Pezzini, P.; Chindris, M. Frequency control of isolated wind and diesel hybrid microgrid power system by using fuzzy logic controllers and PID controllers. In Proceedings of the 11th International Conference on Electrical Power Quality and Utilisation, Lisbon, Portugal, 17-19 October 2011; pp. 1-6.

2. Hunter, R.; Eillot, G. Wind-Diesel Systems: A Guide to Technology and Its Implementation; Cambridge University Press: Cambridge, UK, 1994.

3. Nacfaire, H. Wind-Diesel and Autonomous Energy Systems; Elsevier Science Publishers Ltd: Amsterdam, The Netherlands, 1989.

4. Khelif, A.; Talha, A.; Belhamel, M.; Hadj Arab, A. Feasibility study of hybrid diesel-PV power plants in the southern of Algeria: Case study on ARFA power plant. Int. J. Electr. Power Energy Syst. 2012, 43, 546-553.

5. Sedaghat, B.; Jalilvand, A.; Noroozian, R. Design of a multilevel control strategy for integration of stand-alone wind/diesel system. Int. J. Electr. Power Energy Syst. 2012, 35, 123-137.

6. Palmer, M.; Uehara, T.; Shigenobu, R.; Matayoshi, H.; Senjyu, T.; Datta, M. Suppression of power system voltage and frequency fluctuations by decentralized controllable loads. J. Renew. Sust. Energy 2016, 8, 045905.

7. Hara, R.; Kita, H.; Tanabe, T.; Sugihara, H.; Kuwayama, A.; Miwa, S. Testing the technologies. IEEE Power Energy Mag. 2009, 7, 77-85.

8. Hajizadeh, A.; Golkar, M. Control of hybrid fuel cell/energy storage distributed generation system against voltage sag. Int. J. Electr. Power Energy Syst. 2010, 32, 0142-0615.

9. Nandar, C. Robust PI control of smart controllable load for frequency stabilization of microgrid power system. Renew. Energy. 2013, 56, 16-23.

10. Ali, R.; Mohamed, T.; Qudaih, Y.; Mitani, Y. A new load frequency control approach in an isolated small power systems using coefficient diagram method. Int. J. Electr. Power Energy Syst. 2014, 56, 110-116.

11. Jiayi, H.; Chuanwen, J.; Rong, X. A review on distributed energy resources and microgrid. Renew. Sust. Energy Rev. 2008, 12, 2472-2483. 
12. Howlder, A.; Izumi, Y.; Uehara, A.; Urasaki, N.; Senjyu, T.; Saber, A. A robust $H_{\infty}$ controller based frequency control approach using the wind-battery coordination strategy in a small power system. Int. J. Electr. Power Energy Syst. 2014, 58, 190-198.

13. Pan, I.; Das, S. Kriging based surrogate modeling for fractional order control of microgrids. IEEE Trans. Smart Grid 2015, 6, 36-44.

14. Lee, D.; Wang, L. Small-signal stability analysis of an autonomous hybrid renewable energy power generation/energy storage system part I: Time-domain simulations. IEEE Trans. Energy Convers. 2008, 23, 311-320.

15. Singh, V.; Mohanty, S.; Kishor, N.; Ray, P. Robust $H_{\infty}$ load frequency control in hybrid distributed generation system. Int. J. Electr. Power Energy Syst. 2013, 46, 294-305.

16. Masuta, T.; Yokoyama, A. Supplementary load frequency control by use of a number of both electric vehicles and heat pump water heaters. IEEE Trans. Smart Grid 2012, 3, 1253-1262.

17. Zhang, S.; Mishra, Y.; Shahidehpour, M. Fuzzy-logic based frequency controller for wind farms augmented with energy storage systems. IEEE Trans. Power Syst. 2016, 31, 1595-1603.

18. Manjarres, P.; Malik, O. Frequency regulation by fuzzy and binary control in a hybrid islanded microgrid. J. Mod. Power Syst. Clean Energy 2015, 3, 429-439.

19. Palmer, M.; Tachibana, M.; Senjyu, T.; Funabashi, T.; Saber, A.; Datta, M. Grid stabilization with decentralized controllable loads using fuzzy control and droop characteristics. Int. J. Emerg. Elect. Power Syst. 2014, 15, 357-365.

20. Ronilaya, F.; Miyauchi, H. A load frequency control in an off-grid sustainable power system based on a parameter adaptive PID-type fuzzy controller. Int. J. Emerg. Elect. Power Syst. 2014, 15, 429-441.

21. Datta, M.; Senjyu, T. Fuzzy control of distributed PV inverters/energy storage systems/electric vehicles for frequency regulation in a large power system. IEEE Trans. Smart Grid 2013, 4, 479-488.

22. Han, Y.; Young, P.; Jain, A.; Zimmerle, D. Robust control for microgrid frequency deviation reduction with attached storage system. IEEE Trans. Smart Grid 2015, 6, 557-565.

23. Bevrani, H.; Feizi, M.; Ataee, S. Robust frequency control in an islanded microgrid: $H_{\infty}$ and $\mu$-synthesis approaches. IEEE Trans. Smart Grid 2016, 7, 706-717.

24. Sekhar, P.; Mishra, S. Storage free smart energy management for frequency control in a diesel-PV-fuel cell-based hybrid ac microgrid. IEEE Trans. Neural Netw. Learn. Syst. 2016, 27, 1657-1671.

25. Bevrani, H.; Habibi, F.; Babahajyani, P.; Watanabe, M.; Mitani, Y. Intelligent frequency control in an ac microgrid: Online PSO-based fuzzy tuning approach. IEEE Trans. Smart Grid 2012, 3, 1935-1944.

26. Sa-ngawong, N.; Ngamroo, I. Intelligent photovoltaic farms for robust frequency stabilization in multi-area interconnected power system based on PSO-based optimal Sugeno fuzzy logic control. Renew. Energy. 2015, 74, 555-567.

27. Pan, I.; Das, S. Fractional order fuzzy control of hybrid power system with renewable generation using chaotic PSO. ISA Trans. 2016, 62, 19-29.

28. Vachirasricirikul, S.; Ngamroo, I. Robust LFC in a smart grid with wind power penetration by coordinated V2G control and frequency controller. IEEE Trans. Smart Grid 2014, 5, 371-380.

29. Shankar, G.; Mukherjee, V. Load frequency control of an autonomous hybrid power system by quasi-oppositional harmony search algorithm. Int. J. Electr. Power Energy Syst. 2016, 78, 715-734.

30. Mi, Y.; Fu, Y.; Li, D.; Wang, C.; Loh, P.; Wang, P. The sliding mode load frequency control for hybrid power system based on disturbance observer. Int. J. Electr. Power Energy Syst. 2016, 74, 446-452.

31. Pahasa, J.; Ngamroo, I. PHEVs Bidirectional charging/discharging and SoC control for microgrid frequency stabilization using multiple MPC. IEEE Trans. Smart Grid 2015, 6, 526-533.

32. Yang, J.; Zeng, Z.; Tang, Y.; Yan, J.; He, H.; Wu, Y. Load frequency control in isolated micro-grids with electrical vehicles based on multivariable generalized predictive theory. Energies 2015, 8, 2145-2164.

33. Khooban, M.; Niknam, T.; Blaabjerg, F.; Dragievi, T. A new load frequency control strategy for micro-grids with considering electrical vehicles. Electr. Power Syst. Res. 2017, 143, 585-598.

34. Chaturvedi, D.; Umrao, R.; Malik, O. Adaptive polar fuzzy logic based load frequency controller. Int. J. Electr. Power Energy Syst. 2015, 66, 154-159. 
35. Rana, G.; Umrao, R.; Chaturvedi, D. Automatic generation control with polar fuzzy controller considering generation rate constraint in deregulated power system. In Proceedings of the 2012 International Conference on Advances in Engineering, Science and Management (ICAESM), Nagapattinam, India, 30-31 March 2012; pp. 610-615.

36. Ou, T.; Hong, C. Dynamic operation and control of microgrid hybrid power systems. Energy 2014, 66, 314-323.

37. Hong, C.; Ou, T.; Lu, K. Development of intelligent MPPT (maximum power point tracking) control for a grid-connected hybrid power generation system. Energy 2013, 50, 270-279.

38. Pan, M.; Yan, J.; Tu, Q.; Jiang, C. Fuzzy control and wavelet transform-based energy management strategy design of a hybrid tracked bulldozer. J. Intell. Fuzzy Syst. 2015, 29, 2565-2574.

39. Ortmeyer, T.; Hiyama, T. Frequency response characteristics of the fuzzy polar power system stabilizer. IEEE Trans. Energy Conversion 1995, 10, 333-338.

40. Ou, T.; Lu, K.; Huang, C. Improvement of transient stability in a hybrid power multi-system using a designed NIDC (novel intelligent damping controller). Energies 2017, 10, 488.

41. Ou, T.; Su, W.; Liu, X.; Huang, S.; Tai, T. A modified bird-mating optimization with hill-climbing for connection decisions of transformers. Energies 2016, 9, 671.

42. Moura, A.; Lopes, J.; Moura, A.; Sumaili, J.; Moreira, C. IMICV fault analysis method with multiple PV grid-connected inverters for distribution systems. Electr. Power Syst. Res. 2015, 119, 119-125.

43. Ou, T. A novel unsymmetrical faults analysis for microgrid distribution systems. Int. J. Electr. Power Energy Syst. 2012, 43, 1017-1024.

44. Lin, W.; Ou, T. Unbalanced distribution network fault analysis with hybrid compensation. IET Gen. Tran. Dist. 2011, 5, 92-100.

45. Ou, T. Ground fault current analysis with a direct building algorithm for microgrid distribution. Int. J. Electr. Power Energy Syst. 2013, 53, 867-875.

46. Granizo, R.; Blanquez, F.; Rebollo, E.; Platero, C. A Novel ground fault non-directional selective protection method for ungrounded distribution networks. Energies 2015, 8, 1291-1316.

47. Lotfy, M.; Senjyu, T.; Farahat, M.; Abdel-Gawad, A.; Yona, A. A frequency control approach for hybrid power system using multi-objective optimization. Energies 2017, 10, 80.

48. Das, D.; Roy, A.; Sinha, N. GA based frequency controller for solar thermal-diesel-wind hybrid energy generation/energy storage system. Int. J. Electr. Power Energy Syst. 2012, 43, 262-279.

49. Shimizu, K.; Masuta, T.; Ota, Y.; Yokoyama, A. Load frequency control in power system using vehicle-to-grid system considering the customer convenience of electric vehicles. In Proceedings of the 2010 International Conference on Power System Technology (POWERCON), Hangzhou, China, 24-28 October 2010; pp. 1-8.

50. Senjyu, T.; Nakaji, T.; Uezato, K.; Funabashi, T. A hybrid power system using alternative energy facilities in isolated island. IEEE Trans. Energy Convers. 2005, 20, 406-414.

51. Datta, M.; Senjyu, T.; Yona, A.; Funabashi, T. Minimal-order observer-based coordinated control method for isolated power utility connected multiple photovoltaic systems to reduce frequency deviations. IET Renew. Power Gen. 2010, 4, 153-164.

52. Howlader, A.; Izumi, Y.; Uehara, A.; Urasaki, N.; Senjyu, T.; Yona, A.; Saber, A. A minimal order observer based frequency control strategy for an integrated wind-battery-diesel power system. Energy 2012, 46, 168-178.

53. Umrao, R.; Chaturvedi, D. A novel fuzzy control approach for load frequency control. In Recent Advancements in System Modeling Applications. Lecture Notes in Electrical Engineering; Springer: New Delhi, India, 2013; Volume 188, pp. 239-247.

54. Qudaih, Y.; Ali, R.; Mitani, Y. Microgrid design including fuzzy logic controlled storage system. J. Power Energy Eng. 2014, 2, 374-380.

55. Odeim, F.; Roes, J.; Heinzel, A. Power management optimization of an experimental fuel cell/battery/ supercapacitor hybrid system. Energies 2015, 8, 6302-6327.

56. The Japan Weather Association. Available online: http:/ / www.jwa.or.jp/english (accessed on 28 June 2017).

57. The Okinawa Electric Power Company, Inc. Available online: http://www.okiden.co.jp/en/ (accessed on 28 June 2017).

(C) 2017 by the authors. Licensee MDPI, Basel, Switzerland. This article is an open access article distributed under the terms and conditions of the Creative Commons Attribution (CC BY) license (http:/ / creativecommons.org/licenses/by/4.0/). 